\title{
Synthetic Case Study: Discrimination of Unexploded Ordnance (UXO) and non-UXO Sources with varying Remanent Magnetization Strength using Magnetic Data
}

Wigh, Mark David; Hansen, Thomas Mejer; Døssing, Arne

Published in:

Geophysical Journal International

Link to article, DOI:

10.1093/gji/ggab371

Publication date:

2022

Document Version

Publisher's PDF, also known as Version of record

Link back to DTU Orbit

Citation (APA):

Wigh, M. D., Hansen, T. M., \& Døssing, A. (2022). Synthetic Case Study: Discrimination of Unexploded Ordnance (UXO) and non-UXO Sources with varying Remanent Magnetization Strength using Magnetic Data. Geophysical Journal International, 228(2), 773-791. https://doi.org/10.1093/gji/ggab371

\section{General rights}

Copyright and moral rights for the publications made accessible in the public portal are retained by the authors and/or other copyright owners and it is a condition of accessing publications that users recognise and abide by the legal requirements associated with these rights.

- Users may download and print one copy of any publication from the public portal for the purpose of private study or research.

- You may not further distribute the material or use it for any profit-making activity or commercial gain

- You may freely distribute the URL identifying the publication in the public portal 


\title{
Synthetic case study: discrimination of unexploded ordnance (UXO) and non-UXO sources with varying remanent magnetization strength using magnetic data
}

\author{
Mark David Wigh ${ }^{\circledR},{ }^{1}$ Thomas Mejer Hansen ${ }^{\circledR 2}$ and Arne Døssing ${ }^{1,3}$ \\ ${ }^{1}$ CMAGTRES, Crustal Magnetism Technology and Research Group, DTU Space, Technical University of Denmark, Centrifugevej 356, 2800 Kgs. Lyngby, \\ Denmark.E-mail:mdwi@space.dtu.dk \\ ${ }^{2}$ Department of Geoscience, Aarhus University, Høgh guldbergs-gade 2, 8000 Aarhus C, Denmark \\ ${ }^{3}$ DTU CERE, Center for Energy Resources Engineering, Technical University of Denmark, 2800 Kgs. Lyngby, Denmark
}

\begin{abstract}
SUMMAR Y
We investigate if it is theoretically possible to discriminate between unexploded ordnance (UXO) and non-UXO sources by modelling the magnetic dipole moment for ferrous objects of different shapes and sizes. This is carried out by approximating the volumetric demagnetization factors of rectangular prisms, representing shapes similar to a long rod or flat steel plate. By modelling different UXO as prolate spheroids the demagnetization factors can be determined which can be compared with the magnetic response of a prism. The inversion is carried out in a probabilistic framework, where the UXO forward model and the non-UXO forward model are assigned individual prior models in terms of shape, size, orientation and remanent magnetization of the object. 95 independent realizations of the prism prior model are generated to make 95 synthetic anomalies exemplifying non-UXO objects, which are inverted for using the UXO model. It is investigated if an identical magnetic moment can be produced between the two models and how well resolved the magnetic moment is in terms of the measured anomaly. The case study is carried out in two steps where we first have little prior information of expected UXO properties and another where a UXO prior is introduced with expected values of aspect ratio and size of 24 different UXO, that are often encountered in the North Sea. With no prior information of expected UXO, discrimination is at many times implausible, unless elongated rod prism objects are considered, where the magnetic moment often can not be reproduced by a spheroid. Introducing the UXO prior we achieve a much better discrimination rate when using the list of expected UXO properties. By using the UXO prior we can account for a much higher remanent magnetization allowed in the prior, and still achieve high discrimination capabilities in comparison to a case with no UXO prior.
\end{abstract}

Key words: Magnetic anomalies: modelling and interpretation; Inverse theory; Probability distributions.

\section{INTRODUCTION}

Remote sensing of unexploded ordnance (UXO) by measuring of the magnetic response is the predominant technique in many UXO excavation campaigns. UXO poses major safety hazards in both onshore and offshore zones, hindering productive use of contaminated areas. An efficient and reliable procedure for UXO detection surveys is important for any contractors that have to deal with the possible presence of UXO.

In this study, we seek to discriminate between ferromagnetic objects of different shapes with the aim of characterizing UXO and non-UXO sources using total magnetic intensity (TMI) data. For offshore areas, UXO-clearance is an expensive process and is often done in multiple phases by a combination of TMI and electromagnetic induction (EMI). TMI is usually used as target detection prior to EMI surveys, thereby excluding any possible parametric inversion of the TMI data, which in turn may result in loss of information that could reduce the demand for the more cumbersome EMI surveys. Assuming that UXO generally comprises a ferromagnetic spheroidal or cylindrical shape, we seek to investigate the theoretical possibility of discriminating between UXO and some typical non-UXO objects that are frequently found in offshore areas, such as a long rectangular rod or a flat rectangular plate. 
This study extends the work presented previously in Wigh et al. (2020a), thus having a strong resemblance in the methodology in terms of UXO modelling and probabilistic inversion by Markov chain Monte Carlo (MCMC) sampling. The purpose of this study is to introduce magnetic models of ferromagnetic objects with non-spheroidal shapes, from which we will investigate the theoretical possibility of discriminating between spheroidal and non-spheroidal objects while also accounting for any remanent magnetization present in the sources. The hypothesis is to differentiate between a non-UXO source and a potential UXO (pUXO) source; If we can fit the anomaly signal better by modelling the source as a long or flat rectangular prism than we can with the spheroidal model, then it is highly unlikely that a UXO is causing the anomaly.

The study will be carried out for different cases of prior assumptions of properties describing an UXO. By introducing real quantitative data of found UXO in a seabed survey in the North Sea, a 2-D prior model of the expected volume and aspect ratio is made and used in the inversion. The data consists of 24 different UXO found and enlisted by Ordtek A/S, where the expected aspect ratio and volume of each type are given. A case study where we do not use any prior data of expected UXO will be performed and compared with a case where we introduce the 2-D prior of different UXO as constraint in the inversion. Both cases will be carried out for a varying a priori allowed strength of the maximum allowed remanent magnetization, which ultimately increases the difficulty of discrimination.

\section{METHODOLOGY}

By modelling of rectangular prisms and prolate spheroids, we investigate the difference in the magnetic response from various objects. The focus is explicitly on total field magnetic data, resembling realistic measurements carried out by, for example, either a ship-towed or drone setup using total field magnetometers. Utilizing a probabilistic inversion approach, the likelihood and posterior distribution can be assessed for each prior shape model using MCMC sampling. In this case study, we introduce two conceptual models to be used in the discrimination analysis; the first being the spheroidal forward model, which we assume can effectively reproduce the signal of any UXO. The remaining models are based on a rectangular prism where two objects each with its own prior are defined: a long thin prism and a flat prism. The idea is to approximate the field from either a very long rectangular rod/beam or a flat rectangular plate, each described by unique priors on the two independent aspect ratios that define the prism shape.

\subsection{Magnetic moment}

The magnetic moment of high susceptibility sources can be estimated when the effective susceptibility is known. The effective susceptibility of ferrous sources is dependent on the shape of the object, due to the self-demagnetization effects when ferromagnetic materials are exposed to an external field. The magnetic moment of these objects can be approximated by demagnetization factors, from which the effective or apparent susceptibility can be estimated. The induced dipole moment can be effectively estimated by modelling of a ferromagnetic source using the apparent susceptibility, volume of the source and the orientation of the object. Accounting for any possible remanent magnetization in the ferromagnetic source the dipole moment can be characterized by the following equation (Billings 2006):

$\mathbf{m}_{\text {dipole }}=\frac{V}{\mu_{0}} \mathbf{A}^{T} \bar{\chi}_{d} \mathbf{A} \mathbf{b}_{0}+\mathbf{m}_{\mathrm{rem}}$,

where $\mathbf{m}_{\text {dipole }}$ is the dipole moment of the anomaly, $\mathbf{m}_{\text {rem }}$ is the remanent magnetization of the object, $V$ its volume, $\mathbf{A}(\gamma, \theta, \phi)$ is the Euler rotation matrix, $\bar{\chi}_{d}$ is the effective susceptibilities (a diagonal matrix), and $\mathbf{b}_{0}$ is Earth's magnetic field. $\mu_{0}$ is the permeability of free space. We adopt the three Euler angles as $(\gamma, \theta, \phi)$, where $\gamma$ is the rotation about the spheroidal objects symmetry axis, $\theta$ is the dip angle of the semi-major axis of the spheroid concerning the $x y$-plane and $\phi$ is the azimuthal angle of the semi-major axis relative to true north. This is illustrated in Fig. 1.

For ellipsoidal bodies, the internal demagnetizing field is uniform and the effects thereof can be estimated by calculating the demagnetizing factor, $N$, which is a diagonal tensor along the principal axis of the ellipsoid. For shapes that are non-ellipsoidal, the demagnetizing factor will be a tensor function of position and orientation in the source and thus, cannot simply be assessed by one diagonal tensor. Several studies have been done in determining the magnetometric (volume averaged) demagnetization factors for other non-ellipsoidal shapes, one example being the rectangular prism. These are, in many cases, approximations of the true demagnetization tensor and does not properly express the true nature of the demagnetization tensor for non-ellipsoidal bodies, yet they serve the purpose for tractable approximations of the effective susceptibility.

The apparent or effective susceptibility for a sample with susceptibility $\chi$ and demagnetization factor $N_{i}$ along the principal axis can be calculated by the relation (e.g. Robert \& O’Handley 2000; Dunlop \& Özdemir 2010; Bai et al. 2018):

$\bar{\chi}_{i}=\frac{\chi}{1+N_{i} \cdot \chi}$.

Assuming an isotropic, single element ferromagnetic body the effective susceptibility can be determined once the demagnetization factors are estimated. 


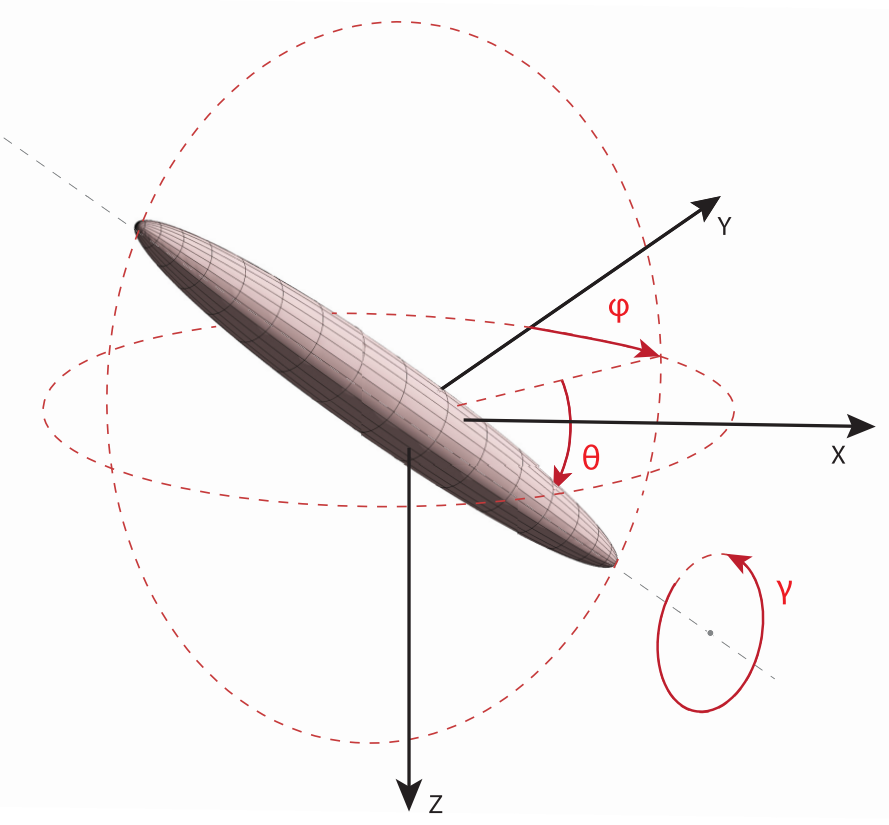

Figure 1. Coordinate system used in the modelling. $Y$-axis aligned with magnetic north, $X$ positive east, $Z$ positive down. The forward model is set up such that the $\phi$ angle is 0 when the semi-major axis of the spheroid/prism is facing towards north, and $\mathbf{m}$ angle is 0 when the spheroid/prism is lying horizontal in the $x y$-plane.

\subsection{Forward model: demagnetization factors of spheroids}

For ellipsoidal bodies the demagnetization factors can be analytically determined. We use the formulation following Beleggia et al. (2006), which exploits the rotational symmetry of the (oblate or prolate) spheroid to effectively determine all three demagnetization factors. In general, the three demagnetization factors are not independent since the trace of the tensor is unity, that is $N_{x}+N_{y}+N_{z}=1$. Thus, if an expression for $N_{z}$ is available we can use the relation $N_{x}=N_{y}=\frac{1}{2}\left(1-N_{z}\right)$. Finally, the expression for $N_{z}^{s}$ is given by

$N_{z}^{s}(e)=\frac{1}{1-e^{2}}\left[1-\frac{e \cdot \arccos (e)}{\sqrt{1-e^{2}}}\right]$

where $e$ is the aspect ratio of the prolate spheroid. Assuming the spheroid has a length, $L$, and diameter, $a$, the aspect ratio is given as the ratio $e=L / a$. Using eq. (3) to determine all three demagnetization factors has shown to be computationally efficient instead of explicitly calculating each factor which has been done previously (Wigh et al. 2020a).

\subsection{Forward model: demagnetization factors of rectangular prism}

The magnetometric demagnetization factors for rectangular prisms have been assessed in several studies (Rhodes 1954; Aharoni 1998; Chen et al. 2002; Beleggia et al. 2006) with the latest study providing exact solutions by utilizing Fourier-Space techniques (Beleggia \& De Graef 2003). In short, it is performed by computing the Fourier representation of the demagnetization tensor field for a given shape of uniform magnetization. This requires that the shape amplitude of the body (e.g. Tandon et al. 2004a, b) can be defined, from which the demagnetization factors can be computed as the volume average of the demagnetization tensor field. The method will not be provided in detail here, but is elucidated in other studies (Beleggia \& De Graef 2003; Beleggia et al. 2005, 2006)

For a given rectangular prism with edge lengths $\left(2 L_{x}, 2 L_{y}, 2 L_{z}\right)$ and volume $V=8 L_{x} L_{y} L_{z}$ the dimensionless aspect ratios are introduced $\lambda_{y}=L_{y} / L_{x}$ and $\lambda_{z}=L_{z} / L_{x}$ and the volumetric demagnetization factor for $N_{z}^{p}$ is Beleggia et al. (2006):

$$
\begin{aligned}
N_{z}^{p}= & G\left(\lambda_{y}, \lambda_{z}\right)=\frac{1}{3 \pi \lambda_{y} \lambda_{z}}\left[-2+\lambda_{y}^{3}+\lambda_{z}^{3}+\left(2-\lambda_{y}^{2}\right) \lambda_{y}^{-}\right. \\
& +\left(2-\lambda_{z}^{2}\right) \lambda_{z}^{-}-\bar{\lambda}_{y z}^{3}+\left(-2+\lambda_{y}^{2}+\lambda_{z}^{2}\right) \Lambda-3 \lambda_{y} \ln \left(\lambda_{y}+\bar{\lambda}_{y}\right)-3 \lambda_{z} \ln \left(\lambda_{z}+\bar{\lambda}_{z}\right)+6 \lambda_{y} \lambda_{z} \arctan \left(\frac{\lambda_{y} \lambda_{z}}{\Lambda}\right)-3 \lambda_{y} \lambda_{z} \ln \left(\lambda_{y}^{\lambda_{y}} \lambda_{y}^{\lambda_{z}}\right) \\
& \left.+3 \lambda_{y}\left(\lambda_{z}^{2}-1\right) \ln \left(\frac{\lambda_{z}}{\lambda_{y}+\Lambda}\right)+3 \lambda_{z}\left(\lambda_{y}^{2}-1\right) \ln \left(\frac{\lambda_{y}}{\lambda_{z}+\Lambda}\right)+3 \lambda_{y} \lambda_{z}^{2} \ln \left(\lambda_{y}+\bar{\lambda}_{y z}\right)+3 \lambda_{y}^{2} \lambda_{z} \ln \left(\lambda_{z}+\bar{\lambda}_{y z}\right)\right]
\end{aligned}
$$


where $\lambda_{y} \equiv \sqrt{1+\lambda_{y}^{2}}, \lambda_{z} \equiv \sqrt{1+\lambda_{z}^{2}}, \lambda_{y z} \equiv \sqrt{\lambda_{y}^{2}+\lambda_{z}^{2}}$ and $\Lambda=\sqrt{1+\lambda_{y}^{2}+\lambda_{z}^{2}}$. Having defined one demagnetization factor of the prism, the remaining two can be computed from eq. (4) by cyclic permutation of the arguments:

$N_{x}^{p}\left(L_{x}, L_{y}, L_{z}\right)=G\left(\lambda_{y}, \lambda_{z}\right)$

$N_{y}^{p}\left(L_{x}, L_{y}, L_{z}\right)=G\left(\frac{\lambda_{z}}{\lambda_{y}}, \frac{1}{\lambda_{y}}\right)$

$N_{z}^{p}\left(L_{x}, L_{y}, L_{z}\right)=G\left(\frac{1}{\lambda_{z}}, \frac{\lambda_{y}}{\lambda_{z}}\right)$.

With the volumetric demagnetization factors defined for rectangular prisms, we can approximate the effective susceptibility for prisms of different shapes using eq. (2). Thus, we can effectively approximate the induced dipole moment of prisms using eq. (1) by sampling of the two aspect ratios, the volume and the orientation of the prism. The volume will be treated as an independent variable, which implicitly defines the size of the prism by the volumetric relation to the demagnetization factors (e.g. $V=8 L_{x} L_{y} L_{z}=8 L_{x}^{3} \lambda_{y} \lambda_{z}$ ).

\subsection{Probabilistic inversion and posterior evidence}

By adopting a probabilistic framework, Bayesian inference can be used to infer what the model parameters of some conceptual model might be, hereby gaining knowledge on quantities of interest that may not be directly observable.

Suppose we have a set of hypotheses (i.e. pUXO/non-UXO models) $\mathcal{H}=\left\{\mathcal{H}_{1}, \ldots, \mathcal{H}_{N}\right\}$ and that for each model, $\mathcal{H}_{i}$, with $i=1, \ldots, N$, is described by a set of parameters $\mathbf{m}_{i}$. And assume that some data (e.g. total-field anomaly data) exist that are related to the model parameters through $\mathbf{d}_{\mathbf{o b s} i}=g_{i}\left(\mathbf{m}_{\mathbf{i}}\right)+n_{i}\left(\mathbf{m}_{\mathbf{i}}\right)$, where $g_{i}$ refer to a specific forward model (either prolate spheroid or prism forward model), and $n_{i}$ refer to a noise model, both related to a specific hypothesis $\mathcal{H}_{i}$. In order to infer what the model parameters, $\mathbf{m}_{i}$, of the hypothesis, $\mathcal{H}_{i}$, might be, given some data, $\mathbf{d}_{\mathbf{o b s}}$. Bayes' theorem can be applied to obtain the posterior probability density (PD), $\sigma\left(\mathbf{m}_{i} \mid \mathbf{d}_{\mathbf{o b s}}, \mathcal{H}_{i}\right)$ of the parameters of interest;

$\sigma\left(\mathbf{m}_{i} \mid \mathbf{d}_{\mathbf{o b s}}, \mathcal{H}_{i}\right)=\frac{p\left(\mathbf{m}_{i} \mid \mathcal{H}_{i}\right) p\left(\mathbf{d}_{\mathbf{o b s}} \mid \mathbf{m}_{i}, \mathcal{H}_{i}\right)}{p\left(\mathbf{d}_{\mathbf{o b s}} \mid \mathcal{H}_{i}\right)}$

The prior PD, $p\left(\mathbf{m}_{i} \mid \mathcal{H}_{i}\right)$, defines the initial state of knowledge before any data has been considered in the inversion phase. The second term in the nominator quantifies the plausibility of the model parameters given the data through the likelihood function; $L\left(\mathbf{m}_{i}, \mathcal{H}_{i} \mid \mathbf{d}_{\mathbf{o b s}}\right) \equiv$ $p\left(\mathbf{d}_{\mathbf{o b s}} \mid \mathbf{m}_{i}, \mathcal{H}_{i}\right)$. The denominator in eq. (5) defines the marginal likelihood, also termed as the evidence (e.g. Sambrigde et al. 2006; Brunetti 2018). It gives a quantitative estimate of the support of observed data to the conceptual model as given by

$p\left(\mathbf{d}_{\mathbf{o b s}} \mid \mathcal{H}_{i}\right)=\int p\left(\mathbf{m}_{i} \mid \mathcal{H}_{i}\right) L\left(\mathbf{m}_{i}, \mathcal{H}_{i} \mid \mathbf{d}_{\mathbf{o b s}}\right) d \mathbf{m}_{i}$.

At the first level of inference, where the posterior distribution of the parameters of one conceptual model is inferred, the evidence is a normalizing constant which usually is neglected (Tarantola 2005; Wigh et al. 2020a). When there are multiple conceptual models and we want to assess the most plausible one given the data, Bayes' theorem can be applied a second time to obtain the posterior probability of the conceptual model $\mathcal{H}_{k}$

$p\left(\mathcal{H}_{k} \mid \mathbf{d}_{\mathbf{o b s}}\right)=\frac{p\left(\mathcal{H}_{k}\right) p\left(\mathbf{d}_{\mathbf{o b s}} \mid \mathcal{H}_{k}\right)}{\sum_{i=1}^{m} p\left(\mathcal{H}_{i}\right) p\left(\mathbf{d}_{\mathbf{o b s}} \mid \mathcal{H}_{i}\right)}$.

When each conceptual model is assumed to have equal prior plausibility, the prior probability, $p\left(\mathcal{H}_{i}\right)$, will be unity and hence eq. $(7)$ will depend solely on the evidence estimates of each conceptual model. Furthermore, if the prior PD of the model parameters are uniform, then the evidence in eq. (6) reduces to the average of the likelihood function over the parameter space of the conceptual model. The evidence can be interpreted as a measure of the volume of space, as defined by the prior distribution, occupied by the posterior distribution. Thus, a very low evidence suggests that locating the posterior distribution is like finding a needle in a haystack (which refer to the space defined by prior distribution). A high evidence, on the other hand, suggests that the prior may be similar to the posterior distribution. The evidence can therefore be seen as a way to quantify the expected computational complexity of sampling the posterior, given a specific choice of prior model. It does, however, not provide any information about the posterior distribution obtained by a specific choice of prior model. Indeed, two hypotheses, with different prior assumptions, can lead to a wildly different evidence, but at the same time, identical posterior distributions. Therefore, we propose an alternative measure of the goodness of a hypothesis with the purpose of risk analysis and decision making, which we refer to as the 'posterior evidence'. The posterior evidence is here defined as the average likelihood of a sample of the posterior, contrary to the evidence, which is computed from a sample of the prior. Say $\mathbf{M}_{\sigma}^{*}=\left[\mathbf{m}_{\sigma}^{*}, \ldots, \mathbf{m}_{\sigma_{N_{r}}}^{*}\right]$ is a sample of $N_{r}$ realizations of $\sigma\left(\mathbf{m} \mid \mathbf{d}_{o b s}, \mathcal{H}_{i}\right)$, then the posterior evidence $(p E)$ can be calculated as

$p E\left(\mathcal{H}_{i}\right)=\frac{1}{N_{r}} \sum_{i}^{N_{r}} L\left(\mathbf{m}_{\sigma_{i}}{ }^{*} \mid \mathbf{d}_{o b s}, \mathcal{H}_{i}\right)$.

The posterior evidence provides a more suitable value for model selection when we are primarily concerned with how good the current model can fit the data at hand, while the complexity of the prior model itself remains of little interest. This is often the case in any UXO 
inversion, since any conceptual model (e.g. a sphere or a prism) that can reproduce the magnetic response equivalently must be included in any risk analysis. For instance, a regular evidence estimate can favour one model over another, even if they can reproduce the same magnetic response, simply because the prior model space for the two conceptual models are different. For further discussion on this, see Appendix A.

The posterior evidence estimates can be used to estimate the relative plausibility of each model given the data at hand. Since we are dealing with synthetic data, the problem can be simplified given that we know the reference models used to produce the different data sets. The likelihood of the reference model is known, and given that we have two hypotheses, non-UXO and pUXO, we compute the likelihood-ratio between the two. In this case study, all data sets are produced by non-UXO (prism) sources which we compare to the posterior evidence achieved using the spheroid forward response. Thus, a likelihood-ratio of 1 shows that no discrimination can be done between the prism and spheroid, while an $L$-ratio closer to 0 indicates that discrimination is more plausible. The likelihood-ratio is defined as the ratio between the posterior evidence of a given hypothesis and the likelihood of the reference model:

$L_{\text {ratio }}\left(\mathcal{H}_{m_{\text {ref }}}, \mathcal{H}_{\mathrm{pUXO}}\right)=\frac{\frac{1}{N_{r}} \sum_{i=1}^{N_{r}}\left(L\left(\mathbf{m}_{i}^{*} \mid \mathcal{H}_{\mathrm{pUXO}}\right)\right)}{\frac{1}{N_{r}} \sum_{i=1}^{N_{r}}\left(L\left(\mathbf{m}_{i}^{*} \mid \mathcal{H}_{m_{\mathrm{ref}}}\right)\right)}=\frac{p E\left(\mathbf{d}_{\mathrm{obs}} \mid \mathcal{H}_{\mathrm{pUXO}}\right)}{p E\left(\mathbf{d}_{\mathrm{obs}} \mid \mathcal{H}_{m_{\mathrm{ref}}}\right)}$,

where $\mathcal{H}_{m_{\text {ref }}}$ refers to the type of prism model (i.e. plate or rod) used to generate the data set and $\mathcal{H}_{\mathrm{puxo}}$ refers to any hypothesis of a potential UXO. In this example, this would be either the complete spheroid prior or a subgroup of it.

As discussed above, we have assumed an uncorrelated noise model. In reality, the noise will be correlated which will lead to scenarios, where using an uncorrelated noise model will result in an apparently very large relative change of likelihood ratio, even though the data response is very similar. In practice, this means most likelihood ratios evaluated using eq. (9) will be 0 or 1 . This may lead to a false conclusion about the resolution power of the method. To alleviate this problem, we make use of a simple annealing method that can be used to mimic a higher noise level, which will lead to more realistic estimates of the likelihood ratio. The amount of annealing is controlled by a 'temperature' parameter, $T$, such that eq. (9) becomes

$L_{\text {ratio (anneal })}\left(\mathcal{H}_{m_{\text {ref }}}, \mathcal{H}_{\mathrm{pUXO}}\right)=\left(\frac{p E\left(\mathbf{d}_{\text {obs }} \mid \mathcal{H}_{\mathrm{pUXO}}\right)}{p E\left(\mathbf{d}_{\text {obs }} \mid \mathcal{H}_{m_{\text {ref }}}\right)}\right)(1 / T)$.

Using $T=1$ eq. (10) will become eq. (9), higher values of $T$ will lead to more annealing (similar to increasing the noise). In the extreme case where $T=\infty, L_{\text {ratio(anneal) }}=1$.

The average likelihood can be evaluated for each model with respect to the data at hand, by MCMC sampling of the posterior distribution. The sampling algorithm used is called Gibbs-Within-Metropolis, which is a combination of the extended metropolis algorithm (Mosegaard \& Tarantola 1995) and Gibbs sampling (Geman \& Geman 1984). In short, it performs a Gibbs step in a randomly selected 2-D model space after $X$ steps have been performed with the extended Metropolis for a better exploration rate in a non-linear model space. This has shown to be an efficient way of sampling the posterior distribution of spheroidal UXO models, as presented in Wigh et al. (2020a), where the algorithm is explained in greater detail.

\subsection{Prior models}

In this case study there will be three conceptual models to express the magnetic response of various ferromagnetic objects; UXO, long rectangular prisms and thin rectangular prisms. All UXO in this example will be modelled as prolate spheroids, whereas the latter two will be approximated by modelling of rectangular prisms. The parametrization of each prism model is essentially the same but with non-identical limits on the prior range of the two aspect ratios defining the shape, thus giving us two unique models that may be used to discriminate in terms of $\mathrm{pUXO} /$ non-UXO objects.

There are nine model parameters shared for all three conceptual models defining the position, orientation, volume of the object and remanent magnetization. These are characterized as $m_{\text {in }}=$ [Depth, Volume, Theta, Phi, X-pos, Y-pos, Mrem Strength, Mrem Incl, Mrem Decl]. The shape of a prolate spheroid is defined from one aspect ratio, $e$, where in terms of rectangular prisms there will be two extra model parameters; one to account for the extra aspect ratio defining the prism (i.e. $\lambda_{y}, \lambda_{z}$ ), and one to account for the rotation along the third axis due to the non-symmetrical shape of the prism, which is redundant for prolate spheroids due to its rotational symmetry. The prior models for all three conceptual models are shown in Table 1, with max-min range specified for all uniform distributions. In Fig. 2 illustrations of the prism objects are seen, as given by the respective ranges of aspect ratio distributions for the rod and plate prior. Keeping the mass fixed at $50 \mathrm{~kg}$ the dimensions are calculated from the max/min values of the aspect ratios, hereby illustrating the range of dimensions allowed for an object with fixed mass. The dimensions can effectively be up-scaled by increasing the mass while keeping the aspect ratios constant.

The UXO prior allows for a larger amount of steel up to $950 \mathrm{~kg}$ and also has a higher remanence of up to 50 per cent, which increases the model complexity and allow for a higher variation in the UXO prior. Since we are focusing only on cases where we can differentiate the magnetic signature of rods and prisms from spheroids, remanent strength for the prism prior is set to a maximum of 5 per cent. This is an arbitrarily low value selected to limit the model variability due to remanence and focus on the induced dipole moment caused by the prism shape.

The depth is constrained to $2.9-4.2 \mathrm{~m}$ in the prior model. The values are chosen to simulate expected distances in actual surveys carried out by techniques such as offshore ship-towed arrays or unmanned aerial vehicle (UAV) systems. The depth prior controls the distance between 
Table 1. Prior models used in the three conceptual models of UXO, rods and plates. The first group of parameters are the globally shared ones that have equal range for each conceptual model, while the last two groups define the unique prior.

\begin{tabular}{ll}
\hline Shared global priors & \multicolumn{1}{c}{ Range (min,max) Uni. Dist. } \\
\hline Depth (z-position) & $2.9-4.2[\mathrm{~m}]$ \\
Phi angle & {$[-180,180]$} \\
Theta angle & {$[-35,35]$} \\
x- position & $\pm 0.5 \mathrm{~m}$ \\
y- position & $\pm 0.5 \mathrm{~m}$ \\
Inclination Mrem & {$[-90,90]$} \\
Declination Mrem & {$[0,360]$} \\
Prism Prior & Range (min,max) Uni. Dist. \\
$\lambda_{y}$ & Rod; $[0.05,0.008]$ Plate; $[1.2,0.82]$ \\
$\lambda_{z}$ & Rod; $[0.005,0.0008]$ Plate; $[0.3,0.004]$ \\
Gamma angle & Rod; $[-90,90] ;$ Plate [-35,35] \\
kg steel & $30-400[\mathrm{~kg}]$ \\
Mrem strength & {$[5]$ per cent of induced moment } \\
Prolate spheroid prior & Range (min,max) Uni. Dist. \\
Aspect ratio, $e$ (Spheroid) & $1.1-7$ \\
kg steel & $30-950[\mathrm{~kg}]$ \\
Mrem strength & {$[(5,50)]$ per cent of induced moment } \\
\hline
\end{tabular}

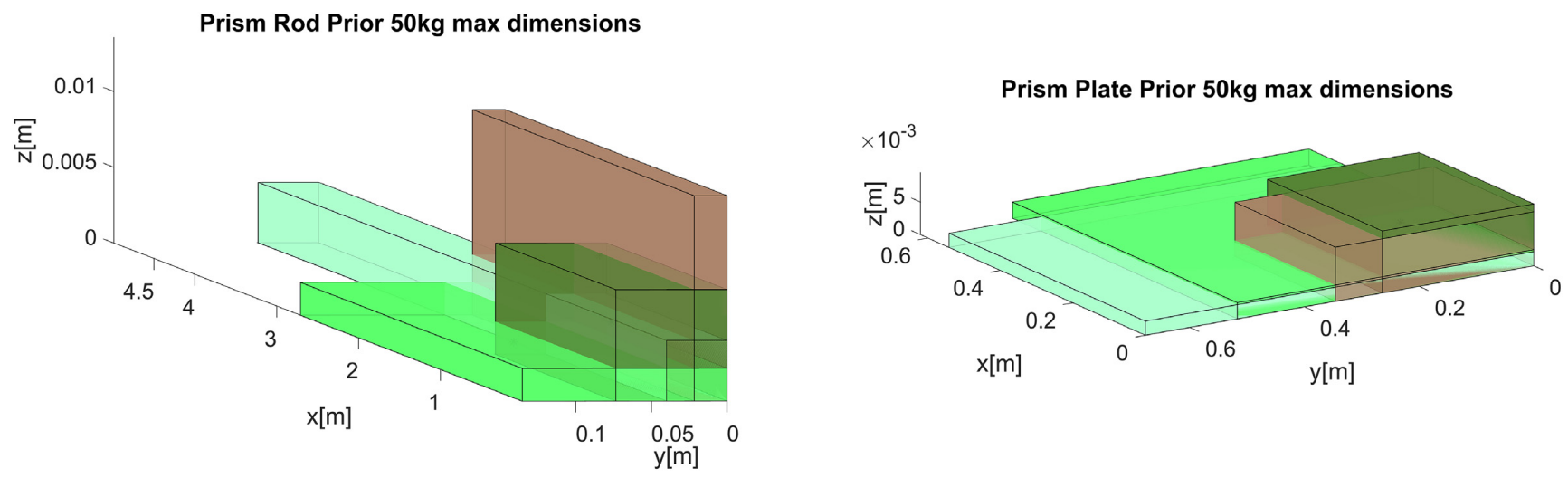

Figure 2. Examples of prism structures created from the prism prior in terms of the prism aspect ratios $\left(\lambda_{y}, \lambda_{z}\right)$. The mass is fixed at $50 \mathrm{~kg}$, showing shapes of the prism model generated from sampling the $\mathrm{max} / \mathrm{min}$ aspect ratios used in the priors. This shows the maximum $/ \mathrm{minimum}$ dimensions of a prism with $50 \mathrm{~kg}$. For larger objects, the dimensions can be up-scaled keeping the aspect ratios constant.

the sensors and source, being an important factor in regards to any expected multipole signals from the modelled source. All induced objects will have some multipole moments that are shape dependent and which signal power falls of rapidly with distance to the source. Modelling the source as a dipole when there are higher order magnetic moments may cause significant errors in the achieved model. The distance should be far enough from the source so multipole moments are non-significant. However, measurements of multipole moments may improve in terms of source discrimination, but this is a more complex manner and will not be discussed in this paper. As a general keynote, the higher order moments are expected to be negligible when the sensor is at a distance of at least 2.5 times the length of the object. As it is evident from Fig. 2, the rod prior may generate simulate very long rods (i.e. 4-7 m length), which are above the distance threshold to neglect higher order moments. In these particular cases when dealing with real data, one would have to account for the presence of higher order moments.

By drawing $X$ samples of the prism prior, we can produce $X$ anomalies from non-UXO sources. The anomalies will then be used as independent data sets where inversion for all three conceptual models will be performed for each anomaly. By calculating the evidence for all cases, we can evaluate the chance of being able to discriminate a rod or plate target from a UXO. This will be carried out for two amplitudes of remanent strength allowed in the UXO prior.

As a final case study, we will include a prior over the aspect ratio and volume of the spheroid model from 24 different UXO found in the North Sea, previously introduced in Wigh et al. (2020b) to improve classification of UXO anomalies in an inversion. This will be used to reduce the model variability of the spheroid model with the purpose of increasing the discrimination chance. The prior data of the UXO types is illustrated in Fig. 3 with 24 different 2-D Gaussian distributions each defined with a relative standard deviation equal to 10 percentage of the volume and 33 percentage of the aspect ratio. In this case, they all have equal probability when integrated over the whole 2-D prior model space. 


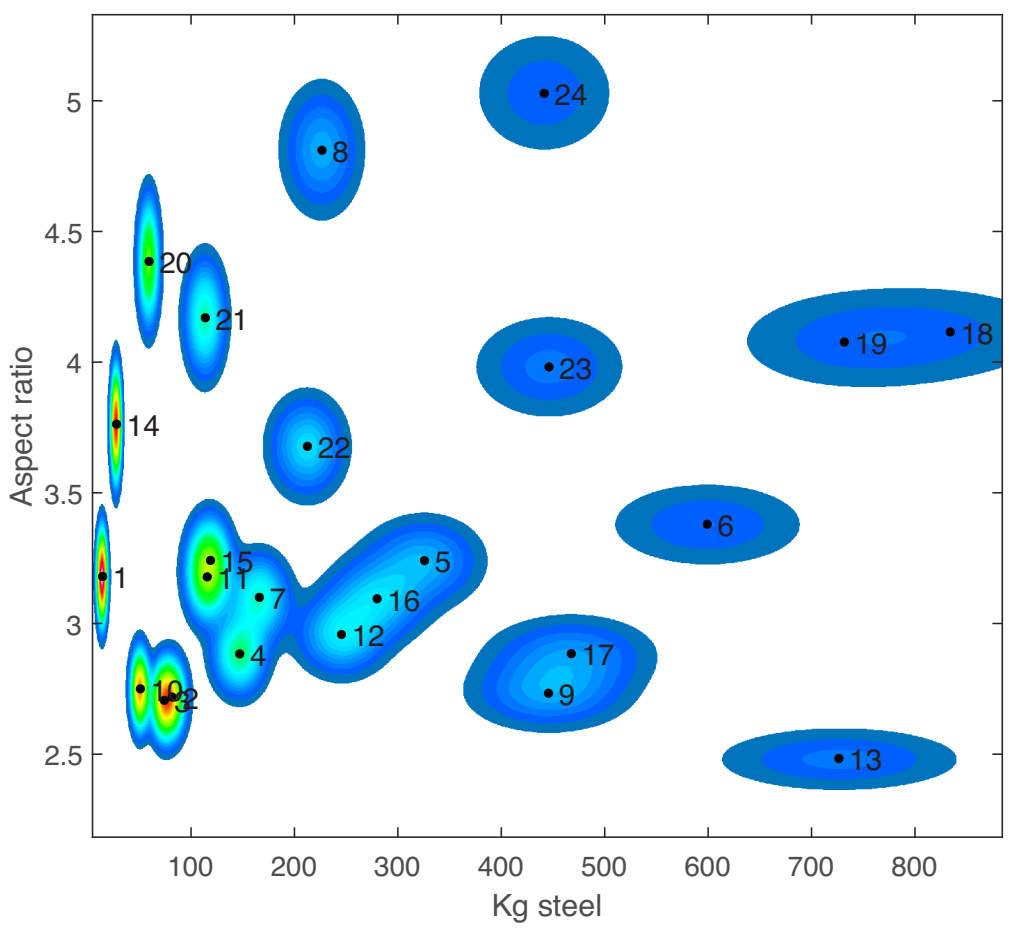

Figure 3. UXO prior model containing properties for 24 different types of UXO. These UXO are found and enlisted by Ordtek Ltd. during UXO excavations in the North Sea. This 2-D prior model will be used as a constraint in terms of aspect ratio and volume in the UXO prior and will be compared to a case where little prior information is available and the two characteristic parameters are uniformly distributed.

\subsection{Sampling setup}

The sampling is in all cases performed using the Gibbs-within-Metropolis algorithm (Wigh et al. 2020a). The sampling setup is different in the cases where we introduce the 2-D prior of expected UXO since we achieve an increase of the constrained model space over the aspect ratio and volume, however, the main concepts are similar. In general, it was apparent, that the UXO inversion often had difficulties converging even if it could perfectly fit the data produced by the prism model. Thus, it was necessary to improve the robustness of the algorithm which was done by making multiple inversions with various setups of the algorithm as well as sampling with an increased temperature.

For the inversions carried out with the full spheroid prior, we use up to a maximum of four independent single-chains. First, an inversion without nearest neighbour interpolation in the Gibbs step of the Gibbs-within-Metropolis sampling algorithm is carried out. If this does fit the data within the noise floor, then we carry out three independent chains with nearest neighbour interpolation enabled. Enabling nearest neighbour interpolation in the Gibbs step allows for a higher exploration rate but less accurate samples, often caused by a sudden jump in the Loglikelihood during sampling. The chain that has the highest mean likelihood after burn-in will then be selected as the final sample. For the case where we introduce the 2-D prior of UXOs, we have $2 \times 24$ independent chains, one for each bomb with and without nearest neighbour interpolation. The two chains should be sufficient since the model space for each of the 24 chains will be far less due to the limitations on aspect ratio and volume, as imposed by the informative prior.

When sampling with low remanent magnetization the iteration limit is set to $150 \mathrm{k}$ metropolis iterations, while sampling with remanent of 50 per cent usually requires more iterations to converge. This iteration limit is set to $700 \mathrm{k}$ and is only carried out for cases where we include the 2-D prior UXO data. Inversion with the full spheroid prior with 5 per cent remanent can be performed within 20 min on a 3-core HPC setup, while sampling using the 2-D prior requires more time in the current setup. This is carried out using 8-cores (3 independent-chains per core) and can be performed in $1 \mathrm{hr}$ and $3.5 \mathrm{hr}$ for 5 and 50 per cent remanent magnetization, respectively.

\section{RESULTS}

The results shown will be focused upon answering the two questions: (1) an we reproduce the same anomaly with the prism prior and the UXO prior, given the prior model assumptions/constraints? (2) Is an anomaly uniquely defined from the moment or how does it differ? We want to investigate the relation between the anomaly measured and the magnetic moment causing the anomaly.

Ninety-five realizations from the prism prior models have been generated and the respective anomaly for each realization have been generated. Out of the 95 random realizations, 53 of these are drawn from the plate prior and the remaining 42 are generated from the rod prior. Each anomaly will be treated as independent data sets originating from 95 different non-UXO objects, generated from samples within the limits of size/shape and orientation /position as specified by the prior model. For each anomaly, we refer to a 'Seed' as being one of the 


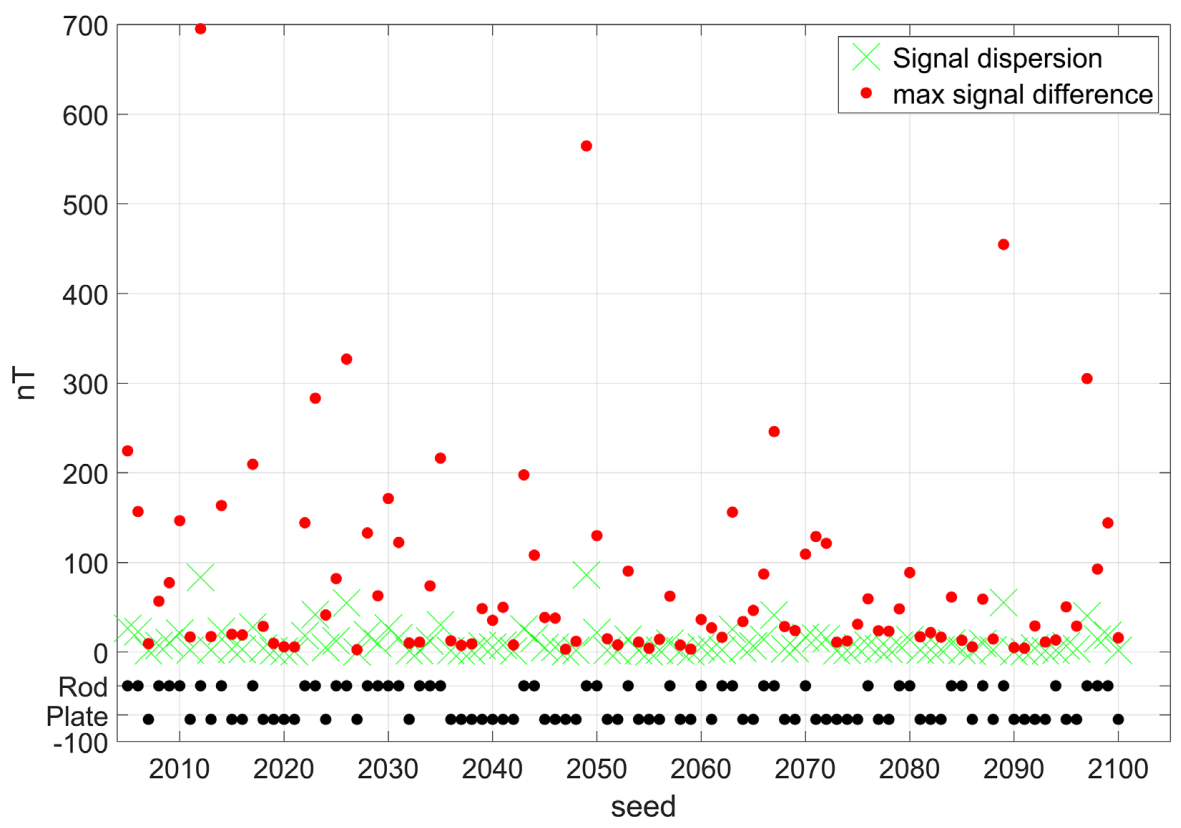

Figure 4. Signal dispersion and $\max /$ min values for all anomalies generated with the prism forward model. Underlying points indicate whether the prior model for a rod or plate has been used to produce the respective prism anomaly.

random realizations of the prism prior, which are used to generate the anomalies. Uncorrelated Gaussian noise is added to every data set independently. By handling the data sets as a 1-D vector we calculate the dispersion of the anomalies as the standard deviation from zero, which is determined as the signal power. The signal-to-noise ratio (SNR) is set to 12 for all data sets. The chosen SNR ratio is arbitrarily selected and might not represent realistic conditions when the signal amplitude of the anomaly is either significantly high or low. However, using the same SNR for all inversions ensures that the sampling difficulty will be somewhat similar for all data sets. For each anomaly, we will perform multiple inversions with the respective prior models in order to determine the fit of the different conceptual models. The result will be carried out in two sections. First, we will perform an inversion example where we only have 1-D prior distributions for the UXO model, and secondly, we will repeat this using the 2-D prior model of UXO size and shape (Fig. 3) for improved inversion resolution.

All anomalies are generated on a $7 \mathrm{~m} \times 7 \mathrm{~m}$ surface with a spatial data resolution of [ $\mathrm{d} x=0.1 \mathrm{~m}, \mathrm{~d} y=0.2 \mathrm{~m}]$. In Fig. 4 the estimated dispersion and $\mathrm{max} / \mathrm{min}$ values of each anomaly is shown. The dispersion is the deviation from zero for a given anomaly and is also what we refer to as signal power. The max/min values are the highest and lowest values of the generated anomalies. It can be seen that the majority of the anomalies generated have a difference in max/min range (i.e. peak to peak values) within 10-100 $\mathrm{nT}$, while approximately 25 of them are above $100 \mathrm{nT}$. It is notable, that all larger anomalies generated with max/min ranges above $100 \mathrm{nT}$ are produced by a rod object. The highest max/min range is around $700 \mathrm{nT}$ and thus has a significantly higher noise added than the smaller anomalies, since the noise is estimated as $1 / 12$ fraction of the dispersion, it will be relative to the anomaly amplitude.

In the following Sections 3.1 and 3.2, we estimate a likelihood-ratio ( $L$-ratio) between the prism forward model and the spheroid forward model. The $\mathrm{L}$ ratio considered is the ratio between the mean Likelihood of a sample of the posterior PD of the spheroid model, and the mean likelihood value given by a sample of the posterior PD associated with the prism reference model. This can be calculated since the reference model and noise in every synthetic data set is known, providing insight into the residual difference between the conceptual models. In Section 3.1 the inversions are carried out over a uniform prior range over the full spheroid prior. In Section 3.2 we introduce the UXO prior containing 24 specific UXO types.

\subsection{Inversion with 1-D uniform spheroid prior}

Inversion of the 95 anomalies is carried out independently using both prism priors, as well as the spheroid prior, according to the prior models shown in Table 1. In this example, the spheroid model utilizes no specific prior over defined UXO types but instead 1-D uniform distributions over the aspect ratio and volume parameters. These are uniformly distributed in ranges that are significantly higher than the range in the UXO prior, as according to the prolate spheroid prior in Table 1. The remaining model parameters are defined as shown in the shared global priors in Table 1 and are identical for all spheroid and prism forward models. In the following section, the achieved likelihood-ratio for inversion of all reference model seeds are shown, together with the relative difference between the mean magnetic moment estimated from inversion with the prism forward and the spheroid forward. In addition, four examples of inversion results and data response will be shown for four selected seeds of the reference models.

The inversions stats are shown in Fig. 5 using the full spheroid prior. From the $L$-ratios it is apparent, that out of the 95 anomalies generated from the prism forward model, $\approx 30$ anomalies can not be fitted equally well by the spheroid forward model, since these ratios are 

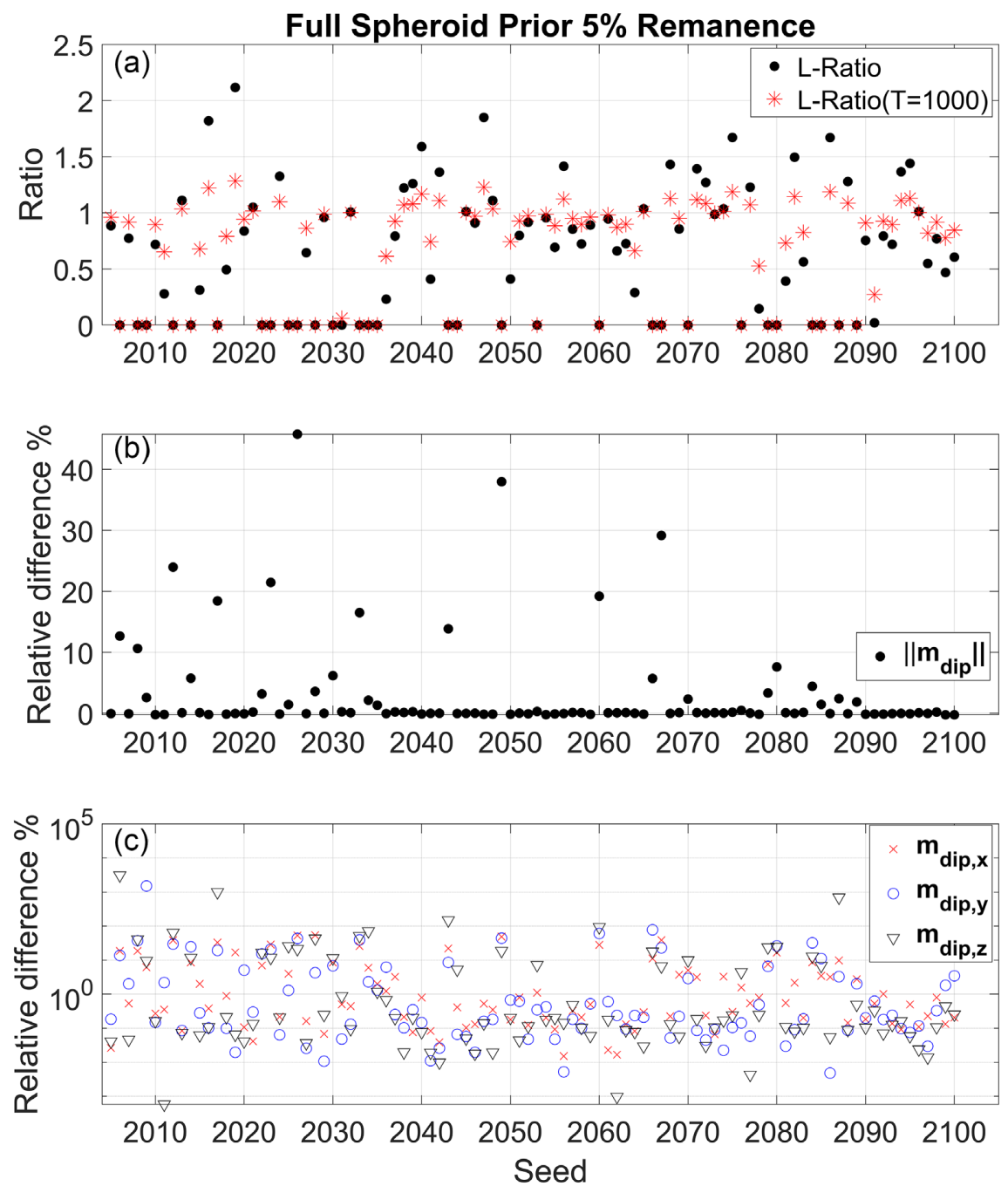

Figure 5. (a) Likelihood ratio between a perfect fit and the mean estimate of the UXO sample from 1-D priors. (b) and (c) Relative difference between the true magnetic moment generated from the conceptual prism model and the average estimated posterior moment for the spheroid model. Panel (b) shows the difference in the euclidean norm of the magnetic moments and panel (c) shows the difference in each of the vector components $(x, y, z)$. A Likelihood ratio of 1 indicates that the spheroid model can fit the data as well as the forward response of the reference model. The likelihood ratio is shown at annealing temperatures of $T=1$ and 1000 .

close to one. An $L$-ratio close to 1 indicates that the data can be fitted, on average, as well as the data response from the reference model fit of the data. In practice then, it will not be possible to discriminate between the possibility of a prism and spheroid from its dipole moment, only based on the fit to the data. Also, as the posterior evidence is the average likelihood of a sample of the posterior, the posterior evidence will be similar using both types of prior, if the $L$-ratio is close to 1 . Because the noise is being added as a function of SNR, anomalies with small amplitudes will have a low and restrictive noise model, and hence, will suggest possible discrimination in cases where the residual field is extremely low. In order to reflect on discrimination chances at higher and more realistic noise levels, the likelihood ratio at an annealing temperature of $T=1000$ is calculated for all cases. The likelihood ratios of 0 indicate that discrimination between a prism and spheroid from its dipole moment is possible in some cases. Figs 5(b) and (c) shows the relative difference between the mean estimate of the dipole moment magnitude and vector components of the prism and spheroid from the posterior samples after burn-in. A positive relative difference of the vector norms indicates a larger magnetic moment of the prism (which is used to generate the anomaly) compared to the achieved moment when inverting with the spheroid model.

Here it is apparent, that the size of the estimated dipole moment is almost identical with a relative difference close to zero in cases where we have an $L$-ratio near 1 . The relative difference between the vector components of the estimated dipole moment is generally low when there is an equal fit. Yet, there are cases where the orientation of the vector is different and we get an equal fit, suggesting that not all anomalies are uniquely defined from the magnetic dipole moment, or that these changes of moment orientation are not affecting our model response significantly in terms of the current spatial resolution and distance to the source. These small differences can also be due to the inclusion of posterior samples before burn-in, or the unlikely event of one of the posterior samples being taken at the point of a Gibbs-iteration jump. These jumps are seen in the four $\log L$ plots, where the sampling with highest mean $\log L$ is achieved using nearest neighbour interpolation in 

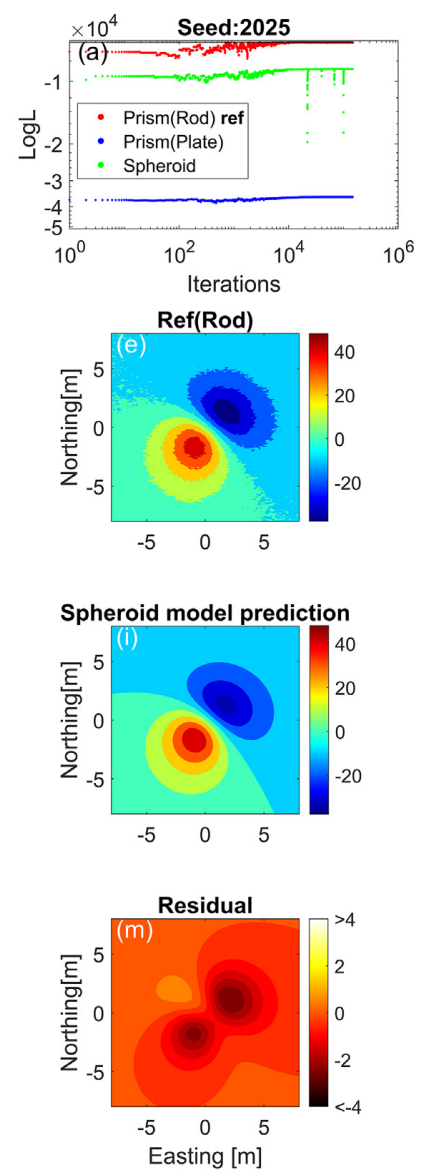

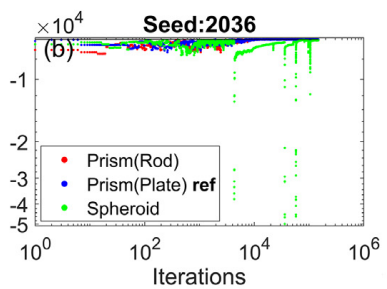

Ref(Plate)
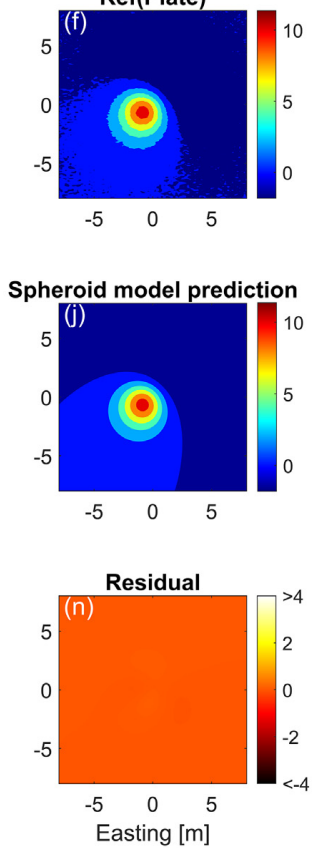
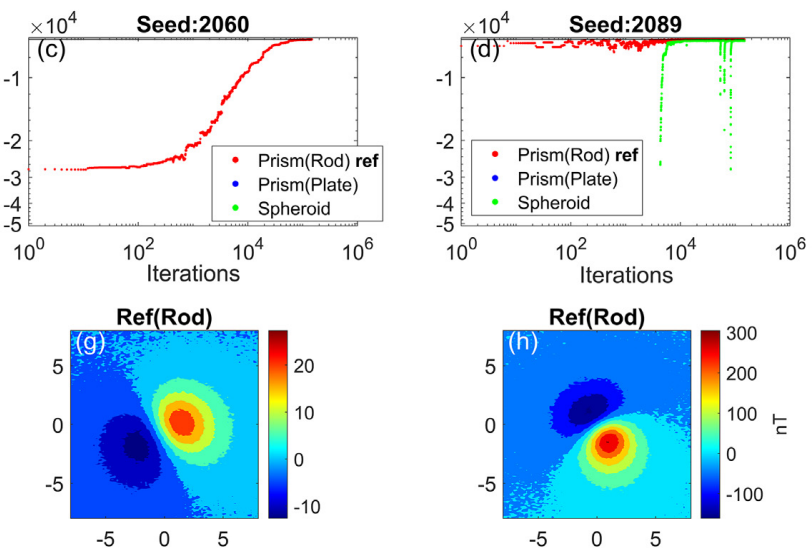

$\operatorname{Ref(Rod)}$
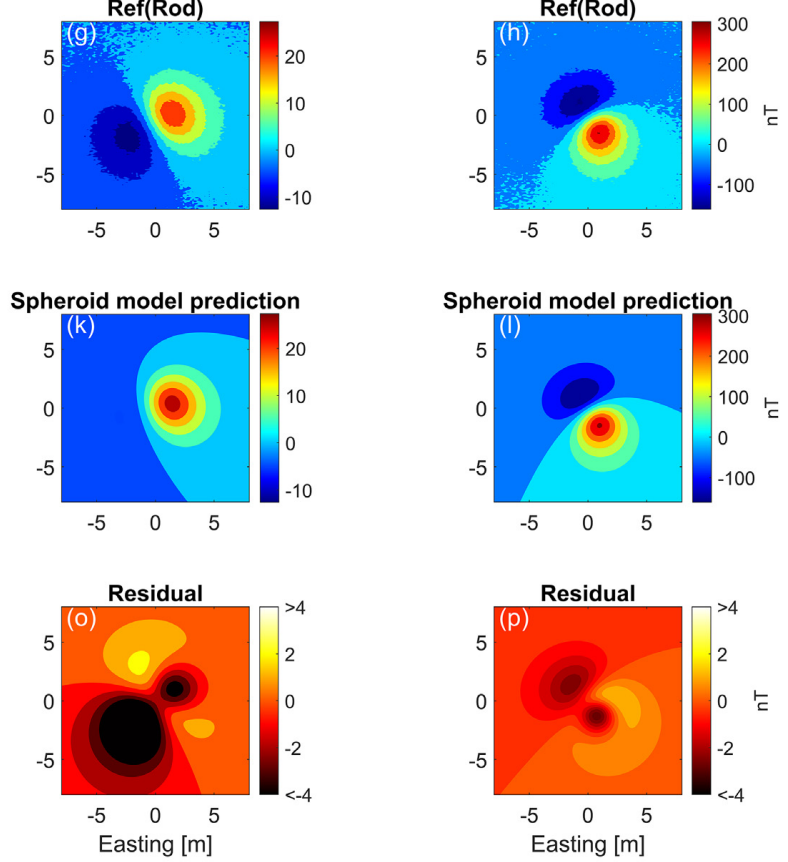

Figure 6. Analysis of inversion of 4 different reference models (one for each column, from left to right seeds shown: [2025, 2036, 2060, 2089]). Top row (a-d): $\log L$ as function of iteration number. Three different inversions are carried out for each reference data set. (Green) Inversion using the spheroid forward and prior. (Red) Inversion with prism forward using the rod prior, and (blue) inversion with prism forward using the plate prior. Second row (e-h): Data set used for inversion, generated using the reference model of the respective seed with uncorrelated noise added. Title indicates which type of prism prior (i.e. Rod or Plate) that is used to generate the anomaly. Third row (i-1): model prediction using the spheroid prior. The fifth last model that has been sampled is used as the model prediction. Fourth row $(\mathrm{m}-\mathrm{p})$ : Residual field between the prism reference model and the model prediction of the spheroid model (noise in the data set is not shown here). The residual field is shown in a different colourmap and a fixed colour scale from -4 to $4 \mathrm{nT}$.

the Gibbs step. These jumps are seen by a sudden, sometimes extreme, jump in $\log L$, but usually followed up by converging back within a short amount of iterations during the metropolis step.

Four examples of the achieved anomaly and residual field between reference data and the UXO estimate can be seen in Fig. 6. Here, we show the $\log L$ during sampling and one example of a model realization from inversion with the prism model, UXO model and the residual field between the two. Each row consider a specific reference model, created using random seeds [2025, 2036, 2060, 2089]. The first row Figs 6(a)-(d) shows the $\log \mathrm{L}$ samples carried out from inversion using the two prism priors, Rod (red) and plate (blue), and the inversion using the spheroid model as shown in green. The second row Figs 6(e)-(h) shows the observed data as created by a realization from either the prism plate or prism rod prior combined with a realization of the noise model. The third row Figs 6(i)-(1) shows the fifth last model sample of the posterior distribution from inversion with the spheroid model, and the last row shows the residual between the observed data and the model prediction of the spheroid prior. The anomalies generated in seed 2036 and 2089 represent cases where we can not discriminate between the spheroid and the prism prior using only the $\log L$ value of realizations from the posterior ( $L$-ratio $\approx 1$ ), while the remaining two, seed 2025 and 2060, are cases where the prism anomaly cannot be fitted equally well using the spheroid model.

For the anomalies represented by seed 2036 and 2089, the prism and spheroid forward response provide similar $\log L$ values of models accepted after burn-in. This can be seen in Figs 6(b) and (d), where the inversion carried out with the spheroid converges to similar values of $\log L$. For seed 2036, being a realization of the plate prior, we note that both two prism priors and the spheroid model converge to similar $\log L$ values. For seed 2089, which is generated from the rod prior, the plate prior does not manage to converge. For the two other cases shown in Figs 6(a) and (c) it is evident, that the spheroid model does not manage to converge to the same value as the prism prior which is used as reference model. A clear residual difference can be seen between the prism and spheroid model for the two seeds in Figs $6(\mathrm{~m})$ and (o). Since the uncorrelated noise added is being scaled with signal power, a higher difference in the $\log L$ results in a higher residual difference due to noise which is relative to the amplitude of the anomaly. 

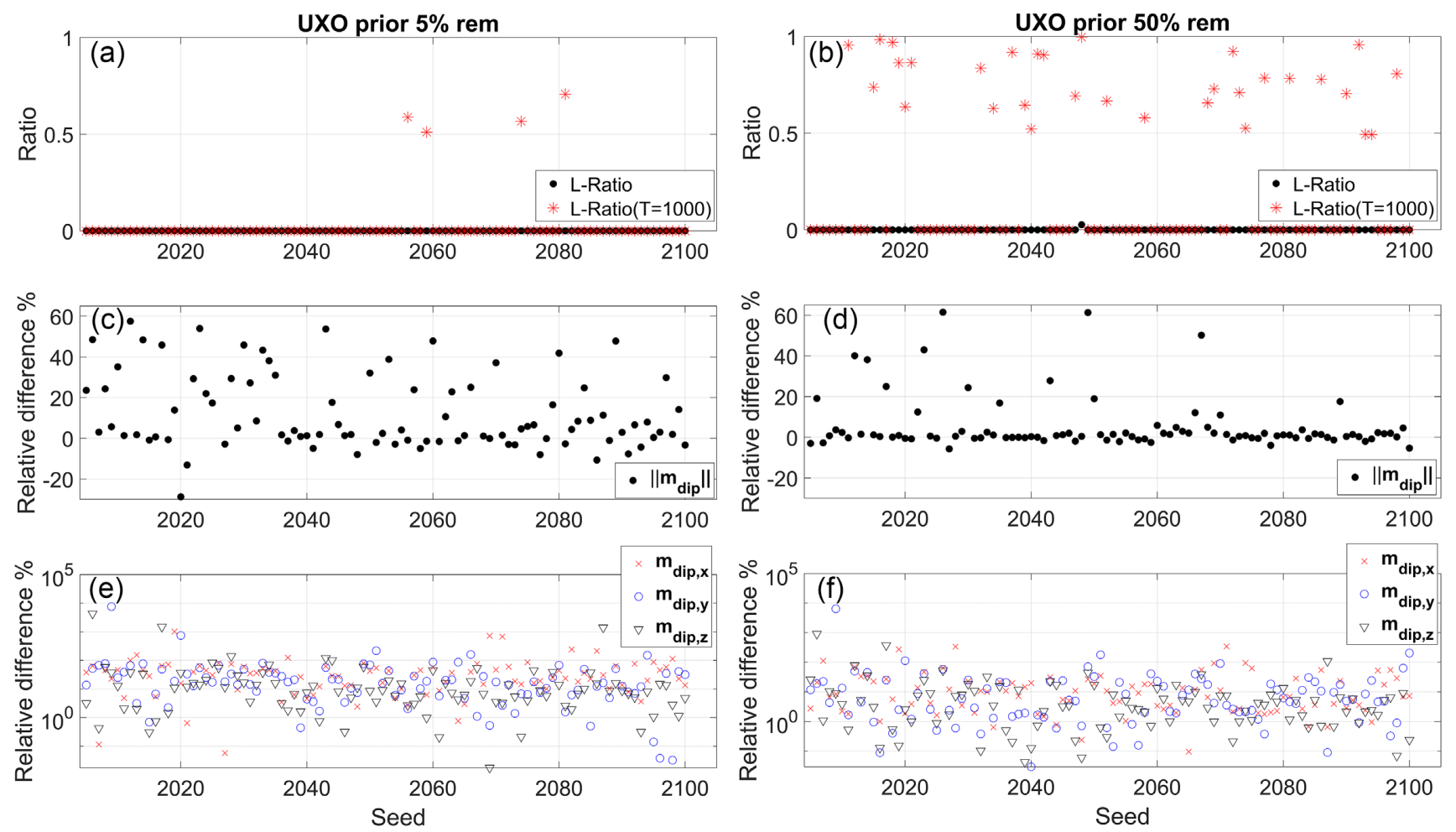

Figure 7. Likelihood ratio between a perfect fit and the mean estimate of the UXO sample using the UXO prior of 24 specified UXO. The Left column shows inversion results with the UXO prior allowing maximum 5 per cent remanent magnetization of the induced moment. In the right-hand column, the same procedure has been carried out but with an allowed remanence of maximum 50 per cent. (a and b) Likelihood ratio between the prism reference model and UXO prior with 5 and 50 per cent remanence, respectively. (c and d) Relative difference between the euclidean norm of magnetic moment generated from the reference prism model and the average estimated posterior moment for the UXO model. Panels (e) and (f) shows the relative difference in each of the vector components $(x, y, z)$. A likelihood ratio of 1 indicates that the UXO prior can fit the data as well as the forward response of the reference model. The likelihood ratio is shown at annealing temperatures of $T=1$ and 1000 .

To ensure that the seeds where we show discrimination success are not due to non-convergence of the algorithm, two examples are made where the inversions are carried out multiple times. For seed 2030 and 2017 we have run the inversion procedure five times (i.e. $5 \times 3$ chains) with different seeds for the random number generator. The chain with the highest mean $\log L$ of each of the five independent $(1 \times 3)$ inversions is selected. For seed 2030 we estimate a mean $\log L$ of $[-8240,-8239,-8247,-8239,-8238]$, and for seed 2017 the estimated $\log L$ values are $[-1.0938,-1.0937,-1.0936,-1.0941,-1.0939] \times 10^{5}$. The mean $\log \mathrm{L}$ achieved from all of the $1 \times 3$ chains differs only slightly and converges to similar $\log L$ values, suggesting that the inversion procedure is robust.

\subsection{Inversion with 2-D UXO prior}

We use the same 95 reference models considered above, but here we invert the reference data using the 2-D UXO prior model, which is more informed than the simple spheroid prior considered above. This has been carried out in two cases, one allowing a maximum remanent magnetization of 5 per cent in the prior, and a second case with 50 per cent maximum remanent magnetization allowed in the prior.

The posterior inversion statistics for both cases of remanent magnetization are shown in Fig. 7. Here, it is seen that in many cases the $\log L$ ratio is far from one, supporting the objective of a higher chance of discrimination when using prior constraints, in terms of expected UXO size and shape. The effects of increasing the remanent magnetization allowed in the prior for the UXO model is apparent in the changes of the estimated $\log L$ ratios. A larger remanent magnetization allows for higher model variability, which will make it easier to reproduce an identical magnetic dipole moment. This is also apparent in the relative differences between the vector norm and vector components. For the case with 5 per cent remanence, we see how the difference of the vector norm is generally higher and correlates well with the low likelihood ratios. There are, however, cases with low $L$-ratios where the difference in the vector norm is small, but where the direction is different as illustrated in the vector component differences.

Four examples of inversions of the reference models are shown in Fig. 8. $\log L$ values, observed data, fitted data and the residual field are shown for the UXO inversion with 5 and 50 per cent remanent magnetization. It is seen how the likelihood generally converges to higher values when sampling with higher remanence and how the residual field reduces in amplitude. For seed 2025 and 2060 in Figs 8 (a) and (c), it is apparent how the inversion with the UXO prior at 5 per cent is far from fitting the anomaly and have $\log L$ values so $\operatorname{low}$ that they are not visible in the plotting range. In all scenarios, it is expected that a higher remanence allowed in the UXO prior would result in a better fit of the signal, given that the UXO prior with low remanence cannot fit the data adequately. Thus, if the inversion with high remanence in the 

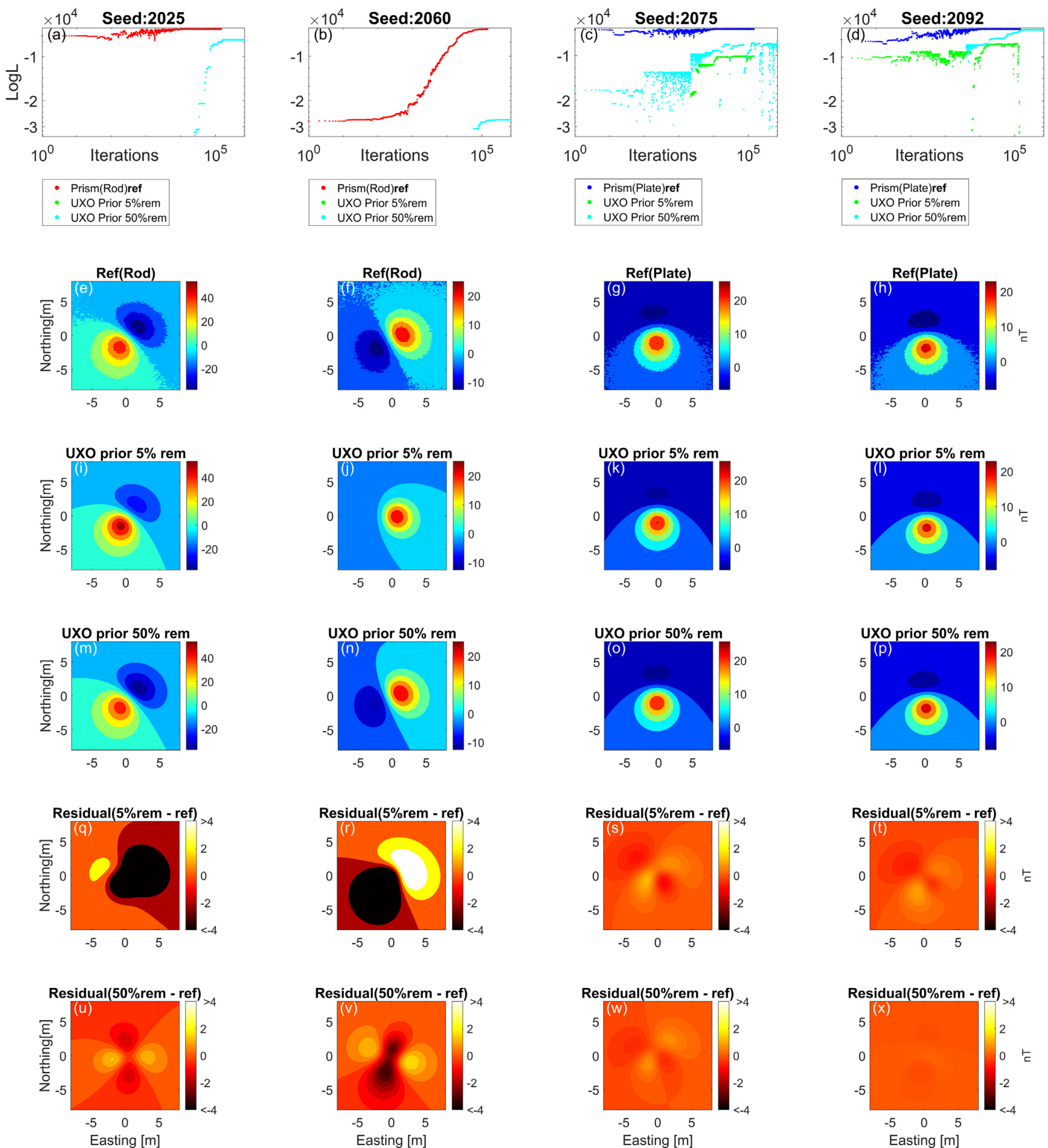

Figure 8. Analysis of inversion of 4 different reference models (one for each column, from left to right seeds shown: [2025, 2060, 2075, 2092]). Top row (a-d): $\log L$ as function of iteration number. Three different inversions are carried out for each reference data set. (Blue/Red) shows the prism prior that is used as reference model. (Green) Inversion using the UXO prior with 5 per cent remanence allowed in the prior. (Cyan) Inversion using the UXO prior with 50 per cent remanence allowed in the prior. Second row $(\mathrm{e}-\mathrm{h})$ : Data set used for inversion, generated using the reference model of the respective seed with uncorrelated noise added. Title indicates which type of prism prior (i.e. Rod or Plate) that is used to generate the anomaly. Third row (i-l): model prediction using the UXO prior with 5 per cent allowed in the prior. Fourth row $(\mathrm{m}-\mathrm{p})$ : model prediction using the UXO prior with 50 per cent allowed in the prior. The fifth last model that has been sampled is used as the model prediction for both cases. Fifth row (q- $\mathrm{t}$ ): Residual field between the prism reference model and the model prediction of the UXO prior with 5 per cent. Sixth row $(\mathrm{u}-\mathrm{x})$ : residual field between the prism reference model and the model prediction of the UXO prior with 50 per cent. The residual field in row five and six are shown in a different colourmap and a fixed colour scale from -4 to 4 nT. 

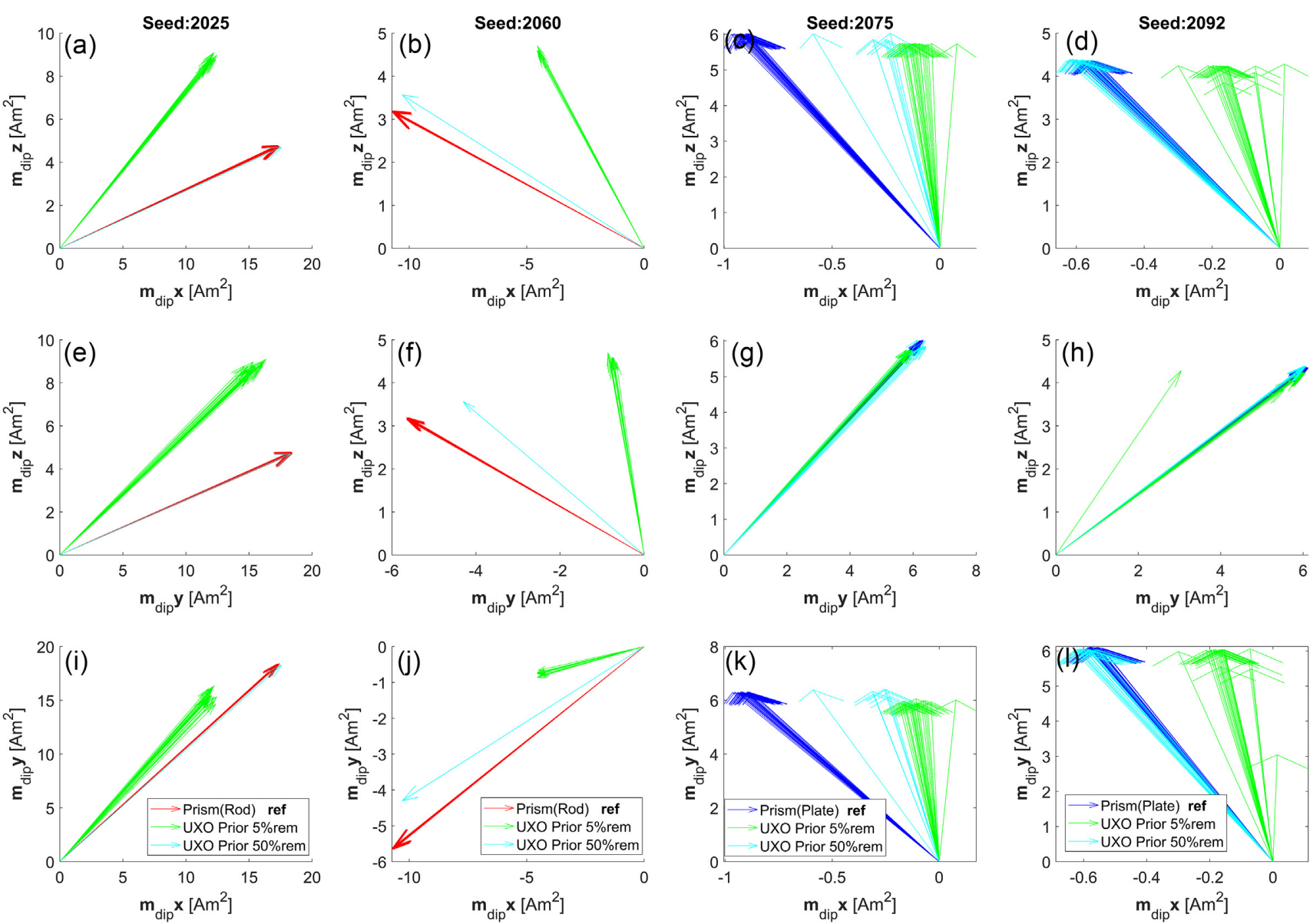

Figure 9. Examples of estimated dipole moment for the three different inversions of the 4 reference models (same as the examples shown in Fig. 8) (One for each column with seeds; [2025, 2060, 2075, 2092]). For every column the three vector components of the dipole moments are shown in the $x z-, y z$ - and $x y$-plane (One for each row, from top to bottom). (Blue/Red) The estimated dipole moment shown for the prism model used as reference. (Green) Dipole moment estimated from inversion with UXO prior with 5 per cent remanence. (Cyan) Dipole moment estimated from inversion with UXO prior with 50 per cent remanence. The last thirty posterior model samples are used to produce the dipole moments in all cases shown.

prior converges to a lower likelihood than a run with low remanence, then this implies that the run with high remanence has not been able to converge properly. Examples of this can be seen in the Likelihood-ratios for seed 2056 and 2059 in Figs 7(a) and (b) where the likelihood ratio for inversion with low remanence is higher than the inversion with high remanence. This is because of the difficulty in converging when sampling with a wide prior for the remanent magnetization strength, suggesting that the inversion procedure might not be robust enough in terms of inversions with high remanence. The robustness of the inversion procedure with high remanence can be improved at the cost of extra computation time. This will be touched upon in the discussion.

In addition, the resulting magnetic moments generated from inversion of the four example seeds are shown in Fig. 9. The first 80 model samples have been removed and then the remaining samples have been used to generate the vector components of the magnetic moment, which are plotted in the $x z-, y z$ - and $x y$-plane. In most cases, the anomaly and magnetic dipole moment is well resolved and uniquely defined in the sense that the anomaly cannot be reproduced by a magnetic moment, that is significantly different in magnitude or direction.

\subsection{Discrimination statistics of prisms and spheroids}

The probability of pUXO/non-UXO are computed using the $L$-ratios for all inversions of the 95 reference models in the three cases (full spheroid prior with 5 per cent rem, UXO prior with 5 per cent rem and UXO prior with 50 per cent rem) are seen in Fig. 10. For each seed, the probability of a non-UXO and pUXO are computed by assuming any non-UXO as prisms and all UXO as spheroids. The probabilities computed from the posterior evidences are similar to the $L$-ratios shown in Figs 5(a), 7(a) and (b) since these calculations are based on the same posterior evidence estimates. Hence, the probabilities of pUXO/non-UXO supports the discrimination chances computed from the $L$-ratios. For this reason, we only show the relative evidence probabilities calculated at an annealing temperature of $T=1000$. In some examples, the spheroid has a higher probability than of the prism, but these are values close to 50 per cent and hence there are zero cases where the object is falsely stated as being a spheroid.

Histograms of the $L$-ratios for all three cases are shown in Fig. 11. The likelihood ratios are here sorted into subgroups of rods and plates. 

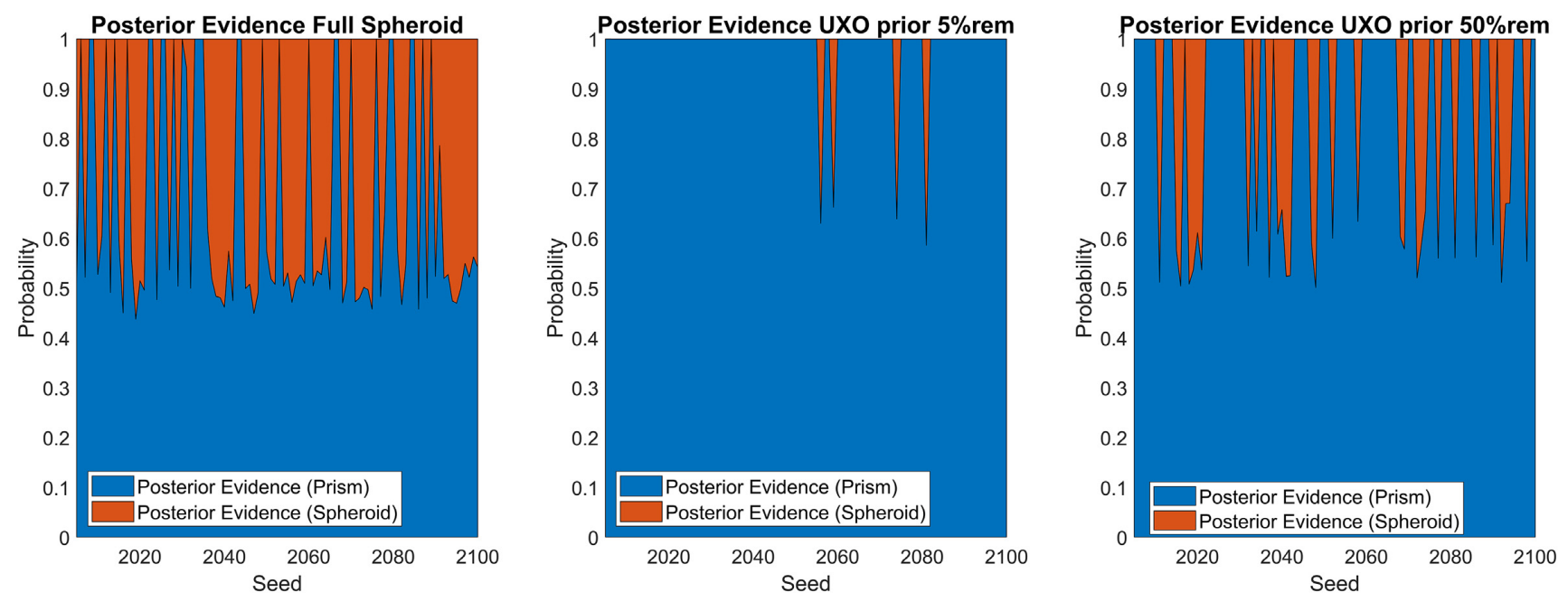

Figure 10. Probability of pUXO/no-UXO computed using the achieved $L$-ratios (with annealing at $T=1000$ ). The relative probabilities are shown for the three inversion cases from left to right) Full spheroid prior, UXO prior with 5 per cent rem and UXO prior with 50 per cent rem. For each seed, the probability of a non-UXO and UXO are computed by assuming any non-UXO as prisms and all UXO as spheroids.
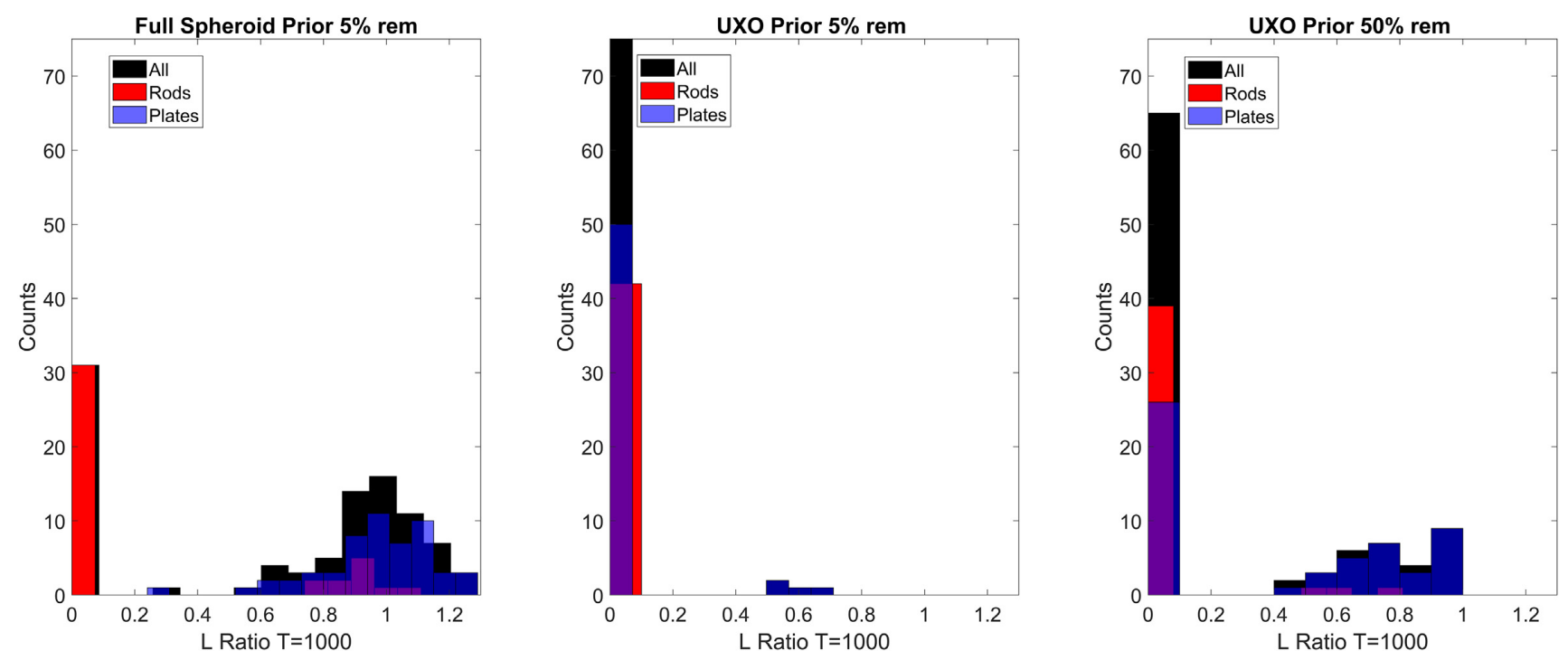

Figure 11. Histogram of likelihood ratios for the three different inversion procedures (Full spheroid prior, UXO prior 5 and 50 per cent remanent magnetization) when performing inversion on the 95 randomly generated anomalies using the prism forward model and the two respective priors. The black histogram represents samples from all cases (rods + plates), and the red and blue are showing the rod and plate anomalies, respectively. An $L$-ratio of 1 indicates that the UXO model can fit the prism anomaly perfectly when considering the noise in the data.

The black histogram shows all $L$-ratios for the 95 data sets, while the red and blue represent a subgroup of these according to which type of prism that has been used to generate the respective data set. By sorting the $L$-ratios in terms of the prism type, it is evident that the plate anomalies (blue histogram) are much more likely to be reproduced by a spheroid compared to anomalies generated from the rod prior (red histogram). It is apparent how the discrimination rate increases when including the UXO prior, and also how a higher remanent magnetization reduces this discrimination rate. Nevertheless, the results carried out with the UXO prior at 50 per cent remanence still provides better discrimination as compared to the case when not using no UXO prior with low remanence. Using the UXO prior increases the discrimination chance for the majority of anomalies produced by rods, while reducing the chance of reproducing the anomalies generated from plates. This illustrates a clear advantage in applying a more informative prior whenever possible.

\section{DISCUSSION}

One important question to consider in this study is, whether the anomaly is uniquely defined from the magnetic dipole moment? It appears in most cases that it is. At least there are no cases where we can reproduce the anomaly with a magnetic moment that is much different from the reference moment. As we see from the relative differences in the components of the magnetic moment and absolute length shown in Figs 5 and 7 a relative difference is usually followed by a difference in the likelihood. 
An important consideration is how much the spatial data resolution affects the estimate of the moment from the measured anomaly, and how well resolved this estimate is. When handling real survey data, one must consider dealing with data of limited resolution where the full anomaly amplitude and signal wavelengths are not captured. This can have consequences on the resolution of the perceived dipole moment and also the position of the source, which again amplifies any ambiguities in the estimated moment.

In cases where we cannot produce the same magnetic moment with the prism and UXO model we will presumably have a misfit (e.g. Likelihood ratio <1) suggesting that we can discriminate between the two. Can we make an $L$-ratio threshold for which we are certain to be able to discriminate? This is very difficult to define since it is affected by factors such as the number of data points, data resolution and noise in the data. In real data knowledge of the noise present in the data is often scarce, although it can be treated as unknown parameters in the inversion, it must be done with caution since a wrong description can lead to very different posterior evidence estimates of the respective conceptual models.

In this study, we have examined if it is possible to distinguish between the magnetic moment of a prism and spheroid by using two different forward models and individual prior model setups. In most cases, it is not possible, but with sufficient prior knowledge, it becomes feasible. When using the full spheroid prior we show discrimination success between prism/spheroid in $\approx 25$ out of 95 cases while we achieve an even higher number using the UXO prior. Most of the 25 cases where we can discriminate with are anomalies produced from the rod prior, while the majority of the plate anomalies can be reproduced by the full spheroid model and hence no discrimination can be made.

The motive for this discrimination procedure relies heavily upon the assumption that all UXO modelled are shaped as prolate (or oblate) spheroids. As it is noted in several studies, the magnetic moment of any ferrous body of arbitrary shape can be reproduced by an ellipsoid, often referred to as the 'Equivalent ellipsoid'. (See Beleggia et al. 2006, fig. 4, where the equivalent ellipsoid is determined as a function of prism aspect ratios.) If any UXO have actual ellipsoidal shapes, an decreased chance of discrimination would be evident. For example, the equivalent ellipsoid of a square cube is a perfect sphere, which would have identical demagnetization factors and induced magnetic moment and hence no discrimination can be done between these two objects.

Considering that our spheroid model contains a uniform distribution of the aspect ratio ranging from 1.1 to 7 , we exclude any possible presence of higher aspect ratios, which might be able to better reproduce the elongated rod objects. In scenarios where objects such as torpedoes are expected one would have to extend the full spheroid range in terms of the aspect ratio, which could reduce the chance of discrimination for anomalies generated from the rod prior.

The fact that there are cases for both rods and plate anomalies, where discrimination is possible, suggests that there can be some discriminative features in the two model spaces. At least for some orientations/shapes of the sources and under the assumption of low remanent magnetization. It is of interest to try and determine areas of the model space where discrimination is possible, however, this would require a thorough analysis of all model parameters in each of the 2-D manifolds. The amount of seeds would have to be increased substantially in order to get enough samples to compare the model spaces. Using the 2-D bomb prior we get significantly better and higher discrimination capabilities. This even works for a much higher remanent magnetization of up to 50 per cent. The success of this returns one to the discussion of using a wrong UXO prior and how devastating it could become if a UXO is falsely classified as a non-UXO. This all comes down to the quality of the prior model. If it is too narrow, it might exclude the true solution, while on the other hand, if it is too wide then we end up with more ambiguities in the model space and our posterior resolution might decrease significantly. These are some of the limitations when performing magnetic modelling from anomaly data, which is why any contribution of knowledge to the prior model is necessary. In this case study, we integrate prior data of UXO types (e.g. as found UXO excavations or history archives), but other remote sensing methods could also be integrated in an effort to gain prior knowledge on parameters related to the depth (if buried) and/or orientation of the object. In offshore seabed surveys, other remote sensing methods such as multibeam echo sounders could be used together or prior to the mag survey, which can help the subsequent inversion if any constraints on orientation can be made. In this study, we have some constraints on the orientation, having the theta angle constrained within $\pm 35^{\circ}$, hereby excluding any possibility of an UXO pointing straight up, for instance. This would be a highly unlikely outcome and excluding this, significantly reduces the amount of ambiguities. In offshore areas with slopes and constant changing topography, it could be a possibility that a UXO or similar have ended in a vertical position. In a real scenario, this would have to be included in a risk assessment for the individual excavation surveys and stakeholders.

Decreasing the spatial data resolution would result in scenarios where anomalies could be reproduced by significantly different dipole moments, hereby reducing any chance of distinguishing between objects of different shapes. In this synthetic example, the spatial resolution of data points is high to ensure a sufficient measurement of the full anomaly within all spatial wavelengths of the signal. The data quality in real surveys is often far less, especially in terms of offshore applications, wherein many cases the full anomaly is not captured, or perhaps masked by other magnetic sources. In such cases, any chance of discrimination may seem unrealistic. However, this serves as a ground study in determining whether it would be feasible to perform such discrimination in the future while also serving as an encouragement for optimizing magnetic survey methods and equipment-setup. A higher resolution of data points will not only increase the discrimination chance but also make it viable to interpret and model correlated noise signals that could originate, for example, from magnetic gradients due to near-surface crustal magnetism. Distance to the source does always need to be considered, both in terms of possible multipole moments but also the loss of signal energy due to objects at large distances. If the target is far away the wavelengths of the measured anomaly will be smaller, which can reduce the accuracy of the estimated dipole moment as well as the positioning accuracy. This is, however, also affected by the spatial resolution of the data set. If the distance between the sensor and object is lower than the size of the body, then multipole moments will be 
significant and will have to be assessed in an inversion. Furthermore, the point-dipole approximation of a large source at low distances will be inaccurate.

There are cases when sampling with the UXO prior, where the low remanent inversion achieves a higher likelihood ratio than the inversion with high remanence. This indicates that the high remanent inversion has not converged properly, even with the increased amount of iterations and total computation time. Clearly, it demonstrates the difficulties in converging when sampling with high remanence which has to be accounted for. To increase this convergence rate one could simply increase the number of independent chains during sampling, or the prior model describing the remanent magnetization could be split up into different intervals (e.g. steps of 10 per cent), each sampled with independent chains. In addition, this could be carried out for each UXO in the prior in order to approximate a marginal variance over the manifold aspect ratio - Volume due to remanent magnetization. This would only have to be carried out once for every bomb, then one would only have to do an inversion using the full spheroid prior which is significantly faster.

\section{CONCLUSION}

In this paper, we have implemented two forward models representing UXO and non-UXO objects to effectively model and interpret magnetic anomalies, with the purpose of discrimination between hazardous UXO and non-UXO targets. The forward models are based on volumetric demagnetization factors calculated from the shape of rectangular prisms and prolate spheroids, from which we can estimate the effective susceptibility that is used to compute the point-dipole moment. We show that with no or little prior information, non-uniqueness is often an issue between the two forward models, in which case, no discrimination can be done. A much better discrimination chance is, however, possible when implementing a prior model that significantly constrains the range on the spheroid model parameters. This has been shown in an example where we introduce a prior of expected UXO in terms of 24 different UXO often encountered in the North Sea. Implementing such informative prior increases the discrimination chance significantly and allows for including a higher remanent magnetization, while still being able to discriminate in many cases. All examples of inversions are carried out on anomaly data with high spatial resolution which is required for a well resolved estimate on the dipole moment, which is necessary for any chance of discrimination.

The methods developed here in terms of probabilistic inversion and model selection can readily be extended to include other models of various shapes as long as the effective susceptibility can be approximated. If one instead seeks to find some threshold of anomaly amplitude in relation to maximum object size then this could simply be applied in the methodology by sampling of the respective prior.

\section{DATA AVAILABILITY}

The data underlying this paper were provided by Ordtek A/S by permission. Data will be shared on request to the corresponding author with permission of Ordtek A/S. The synthetic data generated as part of this paper will be shared on reasonable request to the corresponding author.

\section{REFERENCES}

Aharoni, A., 1998. Demagnetizing factors for rectangular ferromagnetic prisms, J. Phys D: Appl. Phys., 83, 3432, doi:10.1063/1.367113.

Bai, K., Casara, J., Nair-Kanneganti, A., Wahl, A., Carle, F. \& Brown, E., 2018. Effective magnetic susceptibility of suspensions of ferromagnetic particles, J. Phys D: Appl. Phys., 124, $1-12$.

Beleggia, M. \& De Graef, M., 2003. On the computation of the demagnetization tensor field for an arbitrary particle shape using a Fourier space approach, J. Magn. Magn. Mat., 263, L1-L9, doi.org/10.1016/S03048853(03)00238-5.

Beleggia, M., De Graef, M., Millev, Y.T., Goode, D.A. \& Rowlands, G., 2005. Demagnetization factors for elliptic cylinders, J. Phys D: Appl. Phys., 38, 3333-3342.

Beleggia, M., De Graef, M. \& Millev, Y.T., 2006. The equivalent ellipsoid of a magnetized body, J. Phys D: Appl. Phys., 39(5), doi:10.1088/0022$3727 / 39 / 5 / 001$.

Billings, S.D., 2006. Magnetic models of unexploded ordnance, IEEE Trans. Geosci. Remote sens., 44(8), 2115-2124.

Brunetti, C., Linde, N. \& Vrugt, J.A., 2017. Bayesian model selection in hydrogeophysics: application to conceptual subsurface models of the south oyster bacterial transport site, Virginia, USA, Adv. Water Resour., 102, $127-141$.

Brunetti, C., 2018. Bayesian model selection in hydrogeophysics and hydrogeology, PhD thesis, University of Lausanne.

Brunetti, C., Bianchi, M., Pirot, G. \& Linde, N., 2019. Hydrogeological model selection among complex spatial priors, Water Resour. Res., 55(8), 6729-6753, doi.org/10.1029/2019WR024840.
Chen, D.-X., Pardo, E. \& Sanchez, A., 2002. Demagnetizing factors of rectangular prisms and ellipsoids, IEEE Trans. Magnet., 38(4), 1742-1752, doi:10.1109/TMAG.2002.1017766.

Dunlop, D. \& Özdemir, Ö., 2010. Rock Magnetism: Fundamentals and Frontiers (Cambridge Studies in Magnetism), Cambridge Univ. Press.

Robert \& O'Handley, C., 2000. Modern Magnetic Materials: Principles and Applications, John Wiley \& Sons, Inc.

Geman, S. \& Geman, D., 1984. Stochastic relaxation, Gibbs distributions, and the Bayesian restoration of images, IEEE Trans. Pattern Anal. Mach. Intell., 6, 721-741, doi:10.1109/TPAMI.1984.4767596.

Hansen, T.M., Cordua, K.S., Zunino, A. \& Mosegaard, K., 2016. Probabilitsic integration of geoinformation, in Joint Inversion, ed. Moorekamp, M., AGU.

Mosegaard, K. \& Tarantola, A., 1995. Monte Carlo sampling of solutions to inverse problems, J. Geophys. Res., 100(B7), 12 431-12 447, doi.org/10.1029/94JB03097.

Rhodes, P., 1954. Demagnetising energies of uniformly magnetised rectangular blocks, Leeds Philos. Soc., Sci. Sec. Proc., 6, 191-210.

Sambridge, M., Gallagher, K., Jackson, A. \& Rickwood, P., 2006. Trans-dimensional inverse problems, model comparison and the evidence, Geophys. J. Int., 167, 528-542, doi.org/10.1111/j.1365246X.2006.03155.x.

Schöniger, A., Wöhling, T., Samaniego, L. \& Nowak, W., 2014. Model selection on solid ground: rigorous comparison of nine ways to evaluate Bayesian model evidence, Water Resour. Res., 50(12), 9484-9513, doi.org/10.1002/2014WR016062.

Tandon, S., Beleggia, M., Zhu, Y. \& De Greaf, M., 2004. On the computation of the demagnetization tensor for uniformly magnetized particles of 
arbitrary shape. Part I: analytical approach, J. Magn. Magn. Mat., 271(1), 9-26, doi.org/10.1016/j.jmmm.2003.09.011.

Tandon, S., Beleggia, M., Zhu, Y. \& De Graef, M., 2004. On the computation of the demagnetization tensor for uniformly magnetized particles of arbitrary shape. Part II: numerical approach, J. Magn. Magn. Mat., 271, $27-38$.

Tarantola, A., 2005. Inverse Problem Theory and Methods for Model Parameter Estimation, SIAM.
Wigh, M.D., Hansen, T.M. \& Døssing, A., 2020a. Inference of unexploded ordnance (UXO) by probabilistic inversion of magnetic data, Geophys. J. Int., 220, 37-58.

Wigh, M.D., Hansen, T.M. \& Døssing, A., 2020b. Probabilistic inversion of magnetic UXO data: implementing prior UXO data from the North Sea, in Proceedings of the NSG2020 26th European Meeting of Environmental and Engineering Geophysics, European Association of Geoscientists \& Engineers.

\section{APPENDIX: POSTERIOR EVIDENCE}

Say a number of hypotheses exist as $\mathcal{H}_{i}, i=1, \ldots, N$, that reflect different information about some model parameters $\mathbf{m}$. And assume that some data exist that are related to the model parameters through $\mathbf{d}_{i}=g_{i}(\mathbf{m})+n_{i}(\mathbf{m})$, where $g_{i}$ refer to a specific forward model, and $n_{i}$ refer to a noise model, both related to a specific hypothesis $\mathcal{H}_{i}$. Some observed data are available as $\mathbf{d}_{\text {obs }}$.

In a traditional inversion one seek to infer information about the model parameters, as quantified by the posterior probability distribution

$\sigma\left(\mathbf{m} \mid \mathbf{d}_{\mathrm{obs}}, \mathcal{H}_{i}\right)=k^{-1} \rho\left(\mathbf{m} \mid \mathcal{H}_{i}\right) L\left(\mathbf{d}_{\mathrm{obs}} \mid \mathbf{m}, \mathcal{H}_{i}\right)$

$k$ is a normalization constant that ensures that $\int_{\mathcal{M}} \sigma\left(\mathbf{m} \mid \mathbf{d}_{\mathrm{obs}}, \mathcal{H}_{i}\right)=1$, and is also referred to the the evidence and can be interpreted as the probability of a certain hypothesis $\mathcal{H}_{i}$ given some observed data

$P\left(\mathcal{H}_{i} \mid \mathbf{d}_{\text {obs }}\right)$.

The evidence is, for example, used to quantify which of a number of competing hypotheses is most consistent to observed data. The evidence can be computed using several different methods, as discussed in Schöniger et al. (2014). A simple, but computationally costly approach, is to compute the evidence as the average likelihood of a sample of the prior. Say $\mathbf{M}^{*}=\left[\mathbf{m}_{1}^{*}, \ldots, \mathbf{m}_{N_{r}}^{*}\right]$ represents a sample of $N_{r}$ realizations of $\rho(\mathbf{m})$, then the evidence can be estimated as

$E\left(\mathcal{H}_{i}\right)=\frac{1}{N_{r}} \sum_{i}^{N_{r}} L\left(\mathbf{m}_{i}^{*} \mid \mathbf{d}_{\mathrm{obs}}, \mathcal{H}_{i}\right)$.

The relative probability of one, of $N_{h}$, hypotheses is simply given by

$P\left(\mathcal{H}_{i} \mid \mathbf{d}_{\mathrm{obs}}\right)=\frac{E\left(\mathcal{H}_{i}\right)}{\sum_{j=1}^{N} P\left(\mathcal{E}_{j}\right)}$.

A hypothesis can be described through a specific choice of a prior model, in which case analysis of the evidence can be interpreted as quantifying how well a specific choice of prior model is consistent with observed data (e.g. Brunetti et al. 2017, 2019). Below we will challenge this interpretation, through a simple example.

\section{A1 Evidence or 'posterior evidence'?}

Consider a simple 1-D inversion problem. let $m$ refer to a single model parameter. Assume that a data set can be observed, that is simply the value of the model parameter plus normal noise, that is $d=m+n$. The noise model $n$ is assumed be a 1-D normal distribution with zero mean and a standard deviation of 2. The actual value of $m$ is $m_{\text {ref }}=50$, and and observed data is $d_{\text {obs }}=48.7$. The inverse problem is now to infer information about $m$ from $d_{\text {obs }}$.

Four different hypotheses are available, that differ only in their choice of prior model (the forward and the noise is the same for all four cases). Two hypotheses are based on two uniform prior models, $\mathcal{H}_{\text {Uwide }}$ and $\mathcal{H}_{\text {Utight }}$, both consistent with the reference model, in the sense that the reference model has non-zero prior probability. The two uniform models differ only in the range of the uniform distribution, as seen on Fig. A1(a).

Two other hypotheses based 1-D Gaussian prior models are considered as $\mathcal{H}_{\text {Gwide }}$ and $\mathcal{H}_{\text {Gtight }}$, as seen on Fig. A1(a). Note how $\mathcal{H}_{\text {Gtight }}$ is clearly the choice of prior model least consistent with the observed data.

Fig. A1(b) shows the corresponding 1-D posterior distribution for the four considered hypothesis. It is clear the posterior distributions is essentially the same using $\mathcal{H}_{\text {Uwide }}, \mathcal{H}_{\text {Utight }}$ and $\mathcal{H}_{\text {Gwide }}$, and only differs using $\mathcal{H}_{\text {Gtight }}$. In practice this means close to exactly he same information is expressed by the posterior distribution obtained using $\mathcal{H}_{\text {Uwide }}, \mathcal{H}_{\text {Utight }}$ and $\mathcal{H}_{\text {Gwide }}$.

The evidence for each hypothesis is computed using (A3) based on a sample of the prior consisting of 10000 realizations, and shown in Fig. A2(a). It shows that the $\mathcal{H}_{\text {Utight }}$ has much higher evidence than any of the other, and most notably much higher evidence than $\mathcal{H}_{\text {Uwide }}$ In fact

$E($ wide $) / E($ tight $)=2 \times 10^{-45}$.

Yet, at the same time both hypotheses lead to, in practice, the same posterior distribution, Fig. A1(b). 
(a)

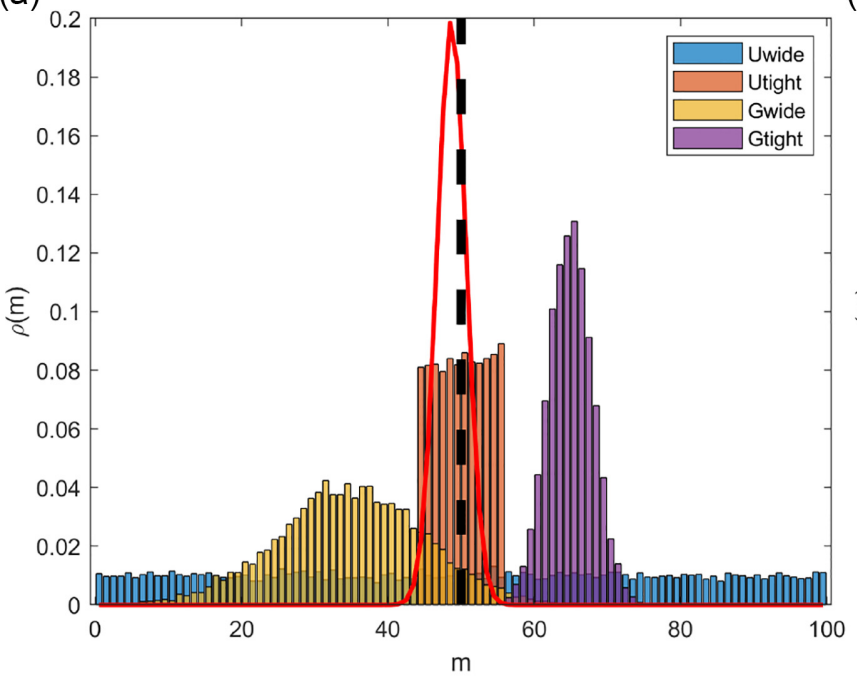

(b)

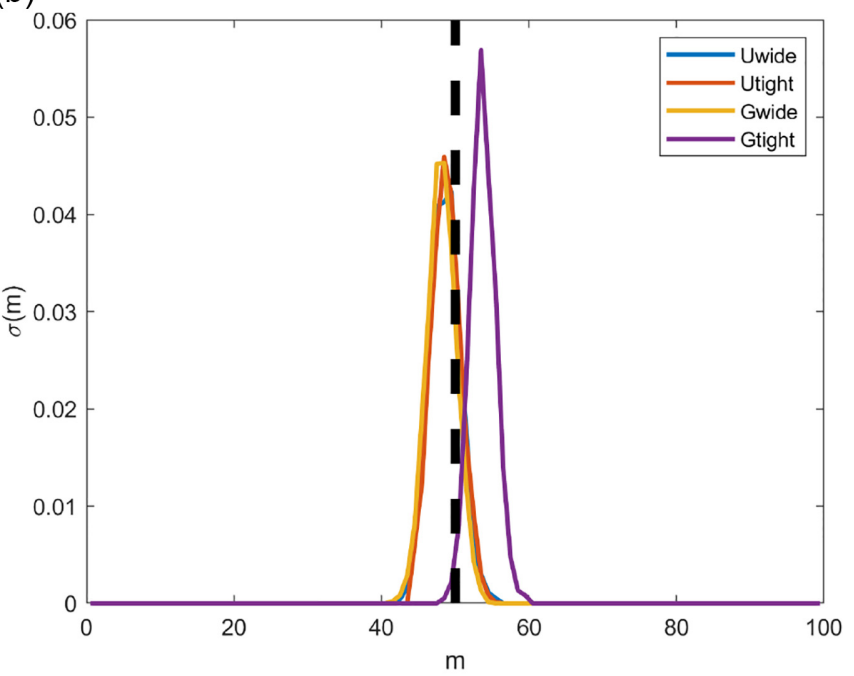

Figure A1. Left-hand panel: 4 examples of prior distributions. Right-hand panel: the corresponding posterior distribution.

(a)

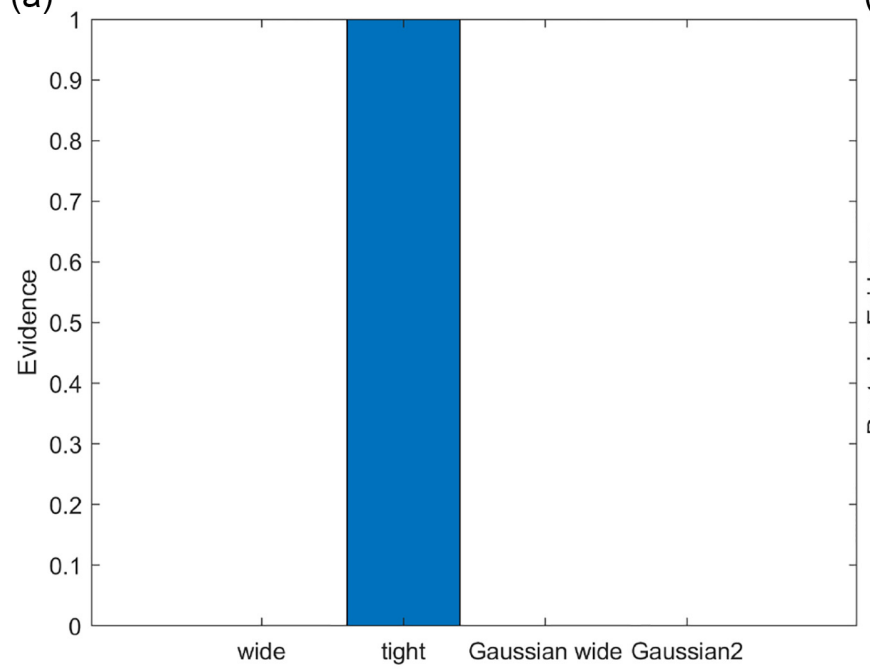

(b)

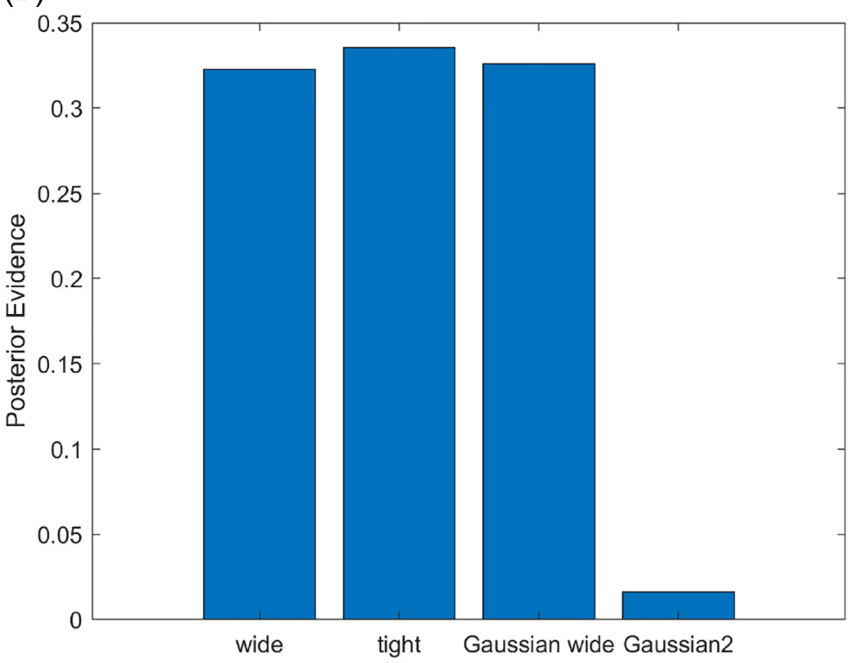

Figure A2. Evidence (a, left), and posterior evidence (b, right), for four hypothesis.

A key point here is that the posterior information provided using $\mathcal{H}_{\text {Utight }}$ and $\mathcal{H}_{\text {Uwide }}$ is, essentially, the same, but that an analysis of the evidence suggests that one choice of hypothesis has wildly higher evidence than the other. From this, we will argue that both choices of prior are equally good given the data, as both results in the same posterior distribution.

Consider another measure we will refer to as the posterior evidence which is defined as the average likelihood of sample of the posterior (as opposed to the average likelihood of a sample for the prior in eq. (A3).

Say $\mathbf{M}_{\sigma}^{*}=\left[\mathbf{m}_{\sigma}^{*}, \ldots, \mathbf{m}_{\sigma}^{*} N_{r}\right]$ is a sample of $N_{r}$ realizations of $\sigma\left(\mathbf{m} \mid \mathbf{d}_{o b s}, \mathcal{H}_{i}\right)$, then the posterior evidence $p E$ can be calculated as

$p E\left(\mathcal{H}_{i}\right)=\frac{1}{N_{r}} \sum_{i}^{N_{r}} L\left(\mathbf{m}_{\sigma}{ }_{i}{ }_{i} \mid \mathbf{d}_{o b s}, \mathcal{H}_{i}\right)$

Fig. A2(b) shows the posterior evidence computed using eq. (A5). The posterior evidence is a measure of the consistency of the hypothesis with the data.

In other words, using $\mathcal{H}_{\text {Utight }}$ leads to as good a fit to data as $\mathcal{H}_{\text {Uwide }}$ and $\mathcal{H}_{\text {Gwide }}$. The evidence suggests that models that fit the data within the noise are located in a much narrower part of the prior model space using $\mathcal{H}_{\text {tight }}$ than using $\mathcal{H}_{\text {wide }}$. But the posterior evidence shows that both can explain the data equally well.

Thus, in terms of decision making, the posterior evidence may be a more useful property than the evidence. Consider two competing hypotheses: $\mathcal{H}_{1}$ (describing an UXO) and $\mathcal{H}_{2}$ (describing a piece of metal/non-UXO). Say an analysis suggest that $E\left(\mathcal{H}_{1}\right)=0.01 E\left(\mathcal{H}_{2}\right)$, but that $P E\left(\mathcal{H}_{1}\right)=0.99 P E\left(\mathcal{H}_{2}\right)$. From the evidence analysis one could conclude that the hypothesis of a UXO is much less probable than that of a non-UXO. On the other hand, the data could be fitted almost equally well by both hypothesis (as quantified by the similar posterior 
evidence), A sample from the two posterior distributions could, as demonstrated above, provide equivalent information. In such a case we would argue that the use of the posterior evidence (average likelihood of a sample of the posterior) is a more useful measure than the evidence.

As the evidence is the average likelihood of a sample of the posterior, the evidence is a precise estimate of the computational efficiency of the extended rejection sampler (a type of rejection sampler that uses the prior as proposal distribution (Hansen et al. 2016) to sample the posterior for a specific hypothesis. It will be wildly inefficient to use the extended rejection sampler to sample the posterior using $\mathcal{H}_{\text {Uwide }}$ rather than $\mathcal{H}_{\text {Utight }}$.

Using the extended Metropolis sampler, the evidence suggests that it may take more computational power (as a larger space of models exist that do not lead to a data fit within the noise) to reach burn-in, that is to locate models with significant likelihood. But once burned in, sampling the posterior using both $\mathcal{H}_{\text {Uwide }}$ rather than $\mathcal{H}_{\text {Utight }}$ will require similar computational demands, as indicated by the similar posterior evidence estimates. 